\title{
Comparisons of Mixing in Chaotic and Turbulent Flows
}

\author{
K. B. SOUTHERLAND, R. D. FREDERIKSEN and W. J. A. DAHM* \\ Gas Dynamics Laboratories, Department of Aerospace Engineering, The University of Michigan, Ann Arbor, \\ MI 48109-2140, USA
}

and

\section{R. DOWLING}

Walter E. Lay Automotive Laboratories, Department of Mechanical Engineering and Applied Mechanics, The University of Michigan, Ann Arbor, MI 48109-2125, USA

\begin{abstract}
Results are presented from an experimental investigation comparing geometric scaling properties created by the mixing of dynamically passive tracers in chaotic flows with those resulting at the small scales of fully developed turbulent flows. The low Reynolds number, two-dimensional, time-periodic, closed flow between eccentric rotating cylinders is taken as the archetypal chaotic flow. The turbulent flow for comparison is the high Reynolds number, three-dimensional, unsteady, open flow in the self-similar far field of a steady axisymmetric jet. For each flow, the concentration field $\zeta(\mathbf{x}, t)$ resulting from the mixing of a conserved scalar quantity is used to measure scaling properties of the support set on which the corresponding scalar energy dissipation rate field $(\operatorname{ReSc})^{-1} \nabla \zeta \cdot \nabla \zeta(\mathbf{x}, t)$ is concentrated. The distributions of dissipation layer separations obtained for both flows are found to be identical. Contrary to central limit arguments for multiplicative quantities, the ensemble-averaged distributions in both flows have a -3 power law scaling for all but the smallest separations; classical log-normal scaling for multiplicative processes is found only in regions having undergone extensive stretching and folding. A statistical assessment of the fractal scaling properties based on one-dimensional intersections with the dissipation support set demonstrates that the chaotic flow at this stage of development approaches a global fractal dimension only in these same regions. Unlike previous studies of the fractal scaling of scalar isosurfaces in turbulent flows, the results for the turbulent flow presented here show no strong evidence for global fractal scaling in the dissipation support set.
\end{abstract}

\section{INTRODUCTION}

Mixing in turbulent flows represents one of the most widely encountered and technically important classes of problems in the fluid sciences. Yet no broadly applicable theory for treating practical problems involving turbulent mixing currently exists. It is known that the quasi-deterministic large scale structures in a turbulent flow control the entrainment properties of the flow, and that these large structures differ from one flow to another. It is believed that the structure and dynamics of the small scales in fully-developed turbulent flows are quasi-universal, satisfying Reynolds number asymptotics and displaying certain universal statistical scaling properties. However, it is only recently that the fully threedimensional, time-varying, small scale structure of mixing in turbulent flows has been accessible to direct experimental study [1]. An example of such a measurement is shown in

\footnotetext{
*Corresponding author.
} 
Fig. 1, where the concentration field $\zeta(\mathbf{x}, t)$ of a dynamically passive, conserved scalar quantity being mixed by the underlying turbulent flow is shown in a small volume element of the flow. The mixing of such a scalar can be usefully quantified in terms of the scalar energy dissipation rate field $(\operatorname{ReSc})^{-1} \nabla \zeta \cdot \nabla \zeta(\mathbf{x}, t)$, also shown for the same volume element in Fig. 1. Of primary interest for the present purposes are the highly folded sheet-like structures into which the scalar dissipation field is formed by the underlying threedimensional time-varying turbulent flow field. It has recently become possible to extract the underlying time-varying vector velocity field $\mathbf{u}(\mathbf{x}, t)$ from such four-dimensional scalar mixing measurements in turbulent flows [2,3]. This may allow detailed studies of the mixing process at the small scales of turbulent flows; however, at present the geometric methods and mathematical tools for describing the structure and dynamics of this mixing process in turbulent flows are still at a relatively early stage of development.

By comparison, considerable progress has been made over the past ten years in the development of experiments and analytical methods for understanding, describing, and even predicting certain features of fluid mixing in closed, two-dimensional, time-periodic chaotic flows and three-dimensional, spatially-periodic, steady flows [4]. Some notable examples of the former class of flows include the blinking vortex flow $[5,6]$, the cavity flow $[4,7,8]$, and the journal bearing flow $[4,7,9]$ shown in Fig. 2. Examples of the latter include the ABC flow [10] and the EHAM flow [11]. Each of these flows, while outwardly simple, produces a periodic stretching and folding of material elements that rapidly manifests itself as an exponential growth in the total amount of intermaterial area available for molecular diffusion. This repeated stretching and folding leads to the formation of highly layered regions of material striations having a wide distribution of separations. With increasing time, these layered regions are further stretched and folded, forming yet smaller striations from initially larger ones via an apparently self-similar multiplicative process.

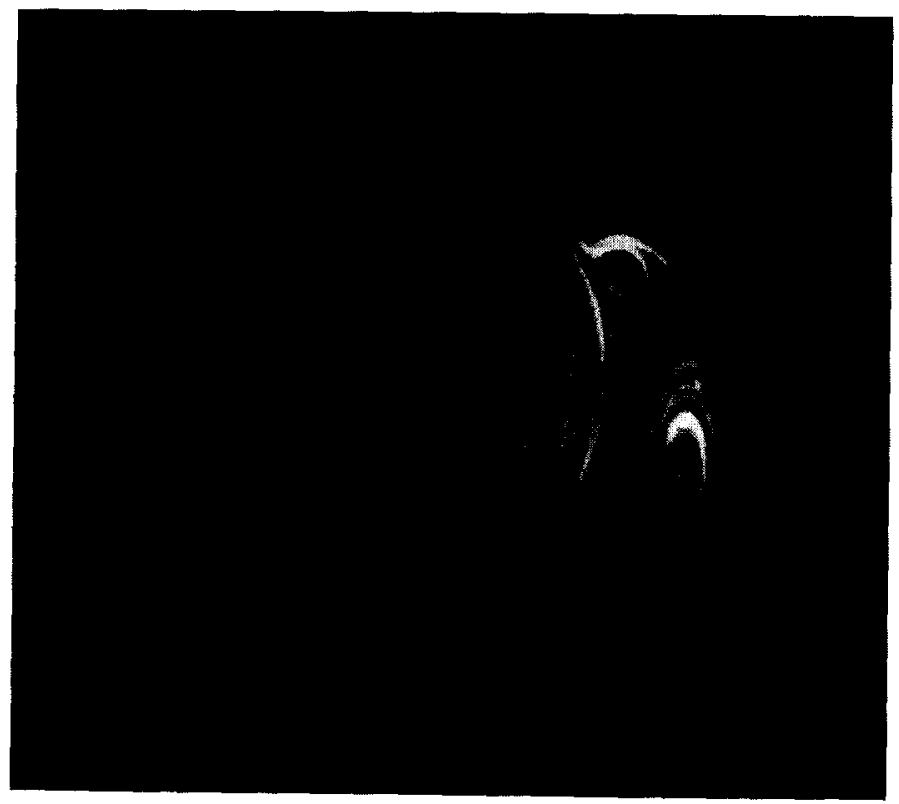

Fig. 2. The scalar field $\zeta(\mathbf{x}, t)$ produced by mixing in the chaotic, two-dimensional, time-periodic eccentric cylinder flow of Swanson and Ottino [12], shown for $\Omega=-3$ and $\vartheta=4 \pi$ at a relatively early period. Note the lamellar structure of the scalar field and compare this with Fig. 1. Shown also are the window boundaries into which the negative is divided for imaging analysis. For reference the boxes are numbered from left to right and top to bottom. 


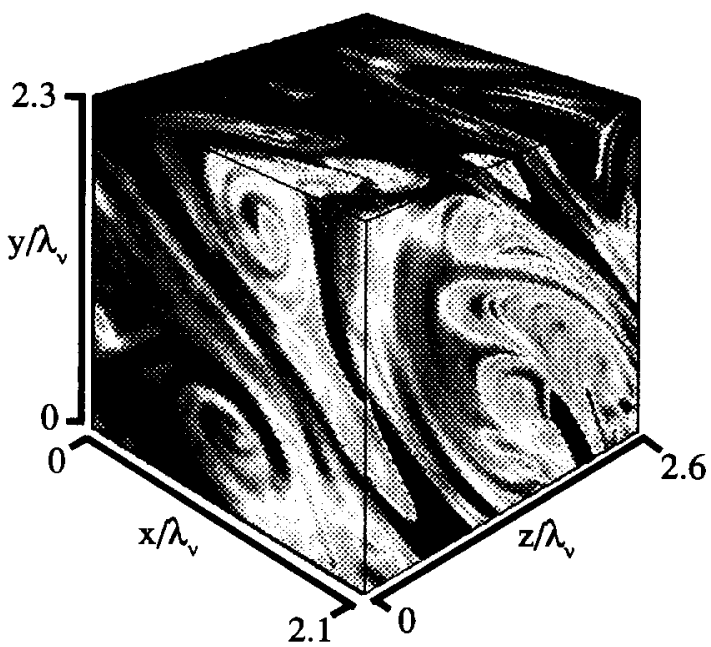

(a)

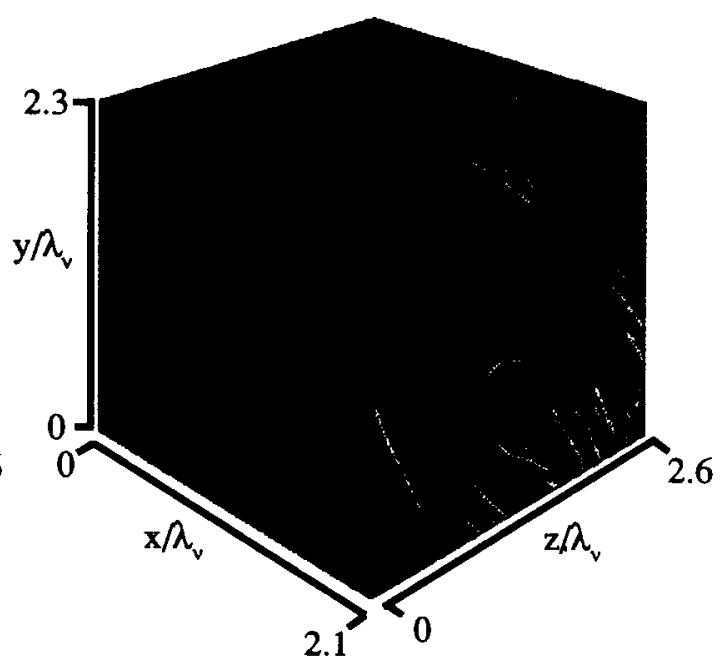

(b)

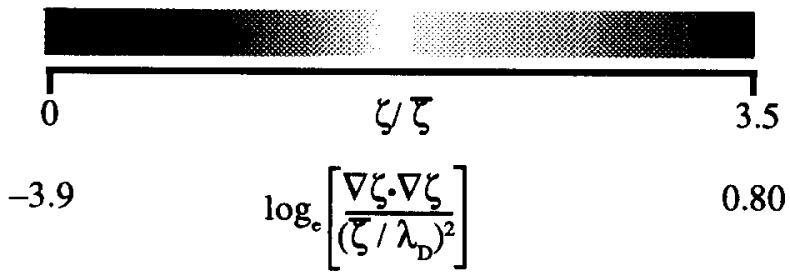

Fig. 1. Experimental data detailing the fluid mixing process at each of over 16 million points in a small three-dimensional spatial data volume taken from a four-dimensional spatio-temporal data space consisting of over 3 billion individual data points obtained in the self-similar far field of an axisymmetric turbulent jet. The volume dimensions are given in terms of the finest velocity gradient length scale $\lambda_{v}$, proportional to the classical Kolmogorov scale. Shown are: (a) the conserved scalar concentration field $\zeta(\mathbf{x}, t)$, and (b) the associated scalar energy dissipation rate $(R e S c)^{-1} \nabla \zeta \cdot \nabla \zeta(\mathbf{x}, t)$. Note the highly convoluted layers in the dissipation field, created by the continual stretching and folding action of the three-dimensional time-varying flow field. For the case shown, $R e \approx 3700$ and $(R e S c) \approx 8 \times 10^{6}$. 
(a)

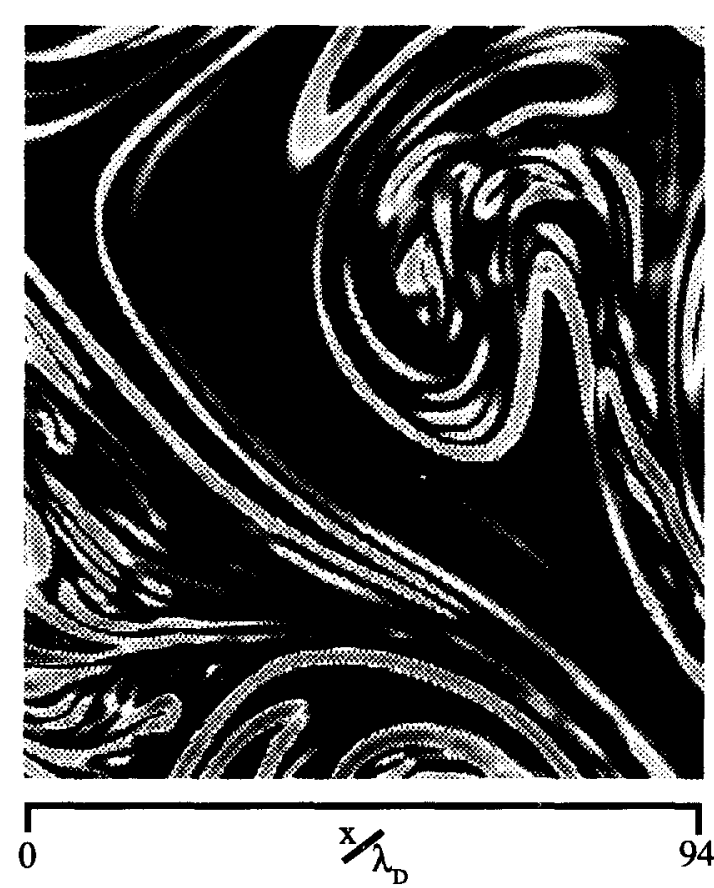

(b)

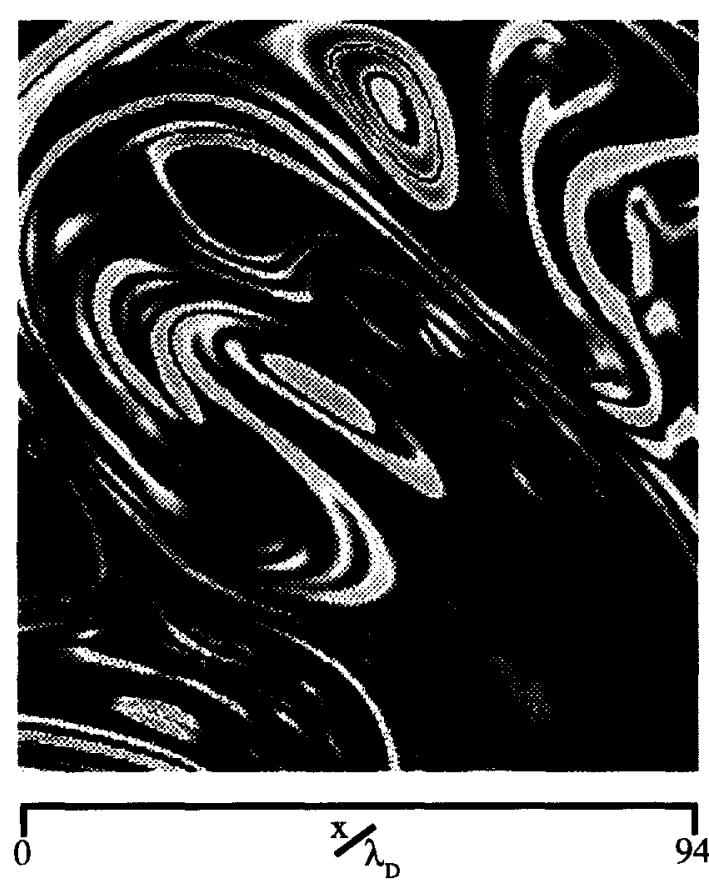

$\Gamma 105$
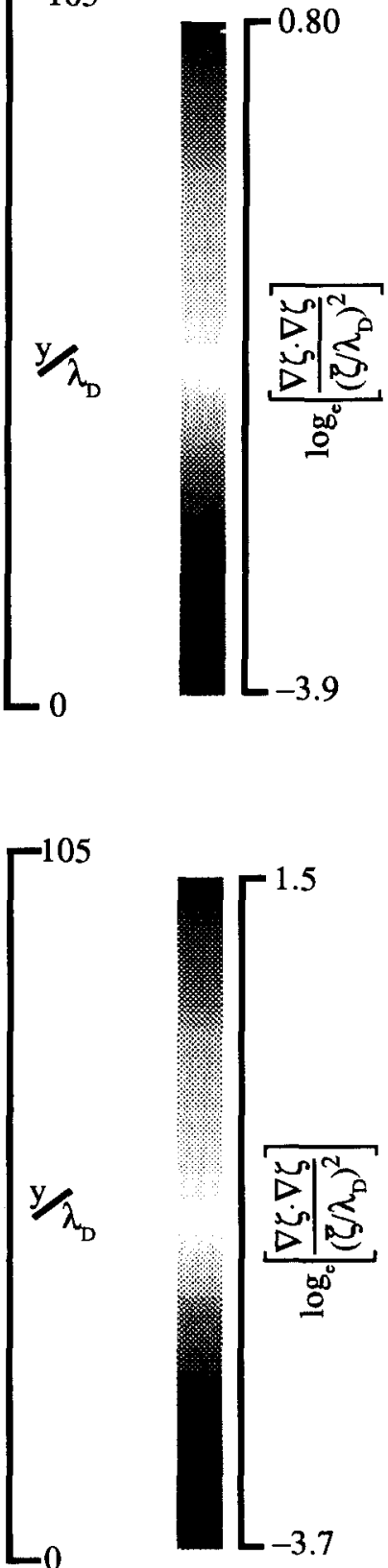

Fig. 3. Examples of the three-dimensional scalar energy dissipation rate field $(\operatorname{ReSc})^{-1} \nabla \zeta \cdot \nabla \zeta(\mathbf{x}, t)$ displayed as two-dimensional cuts from different three-dimensional spatial data volumes of the type shown in Fig. 1. Notice the highly convoluted nature of the layers forming the dissipation field. 
(a)
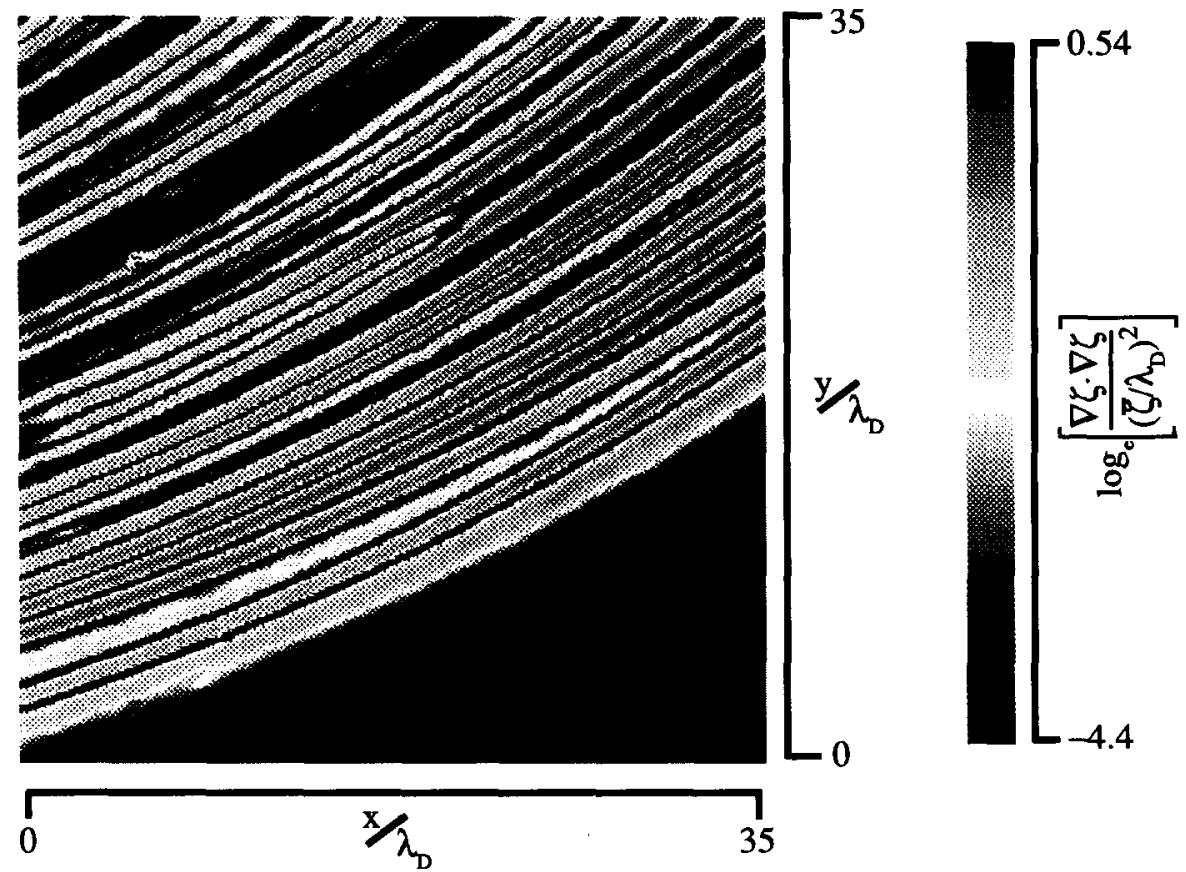

(b)
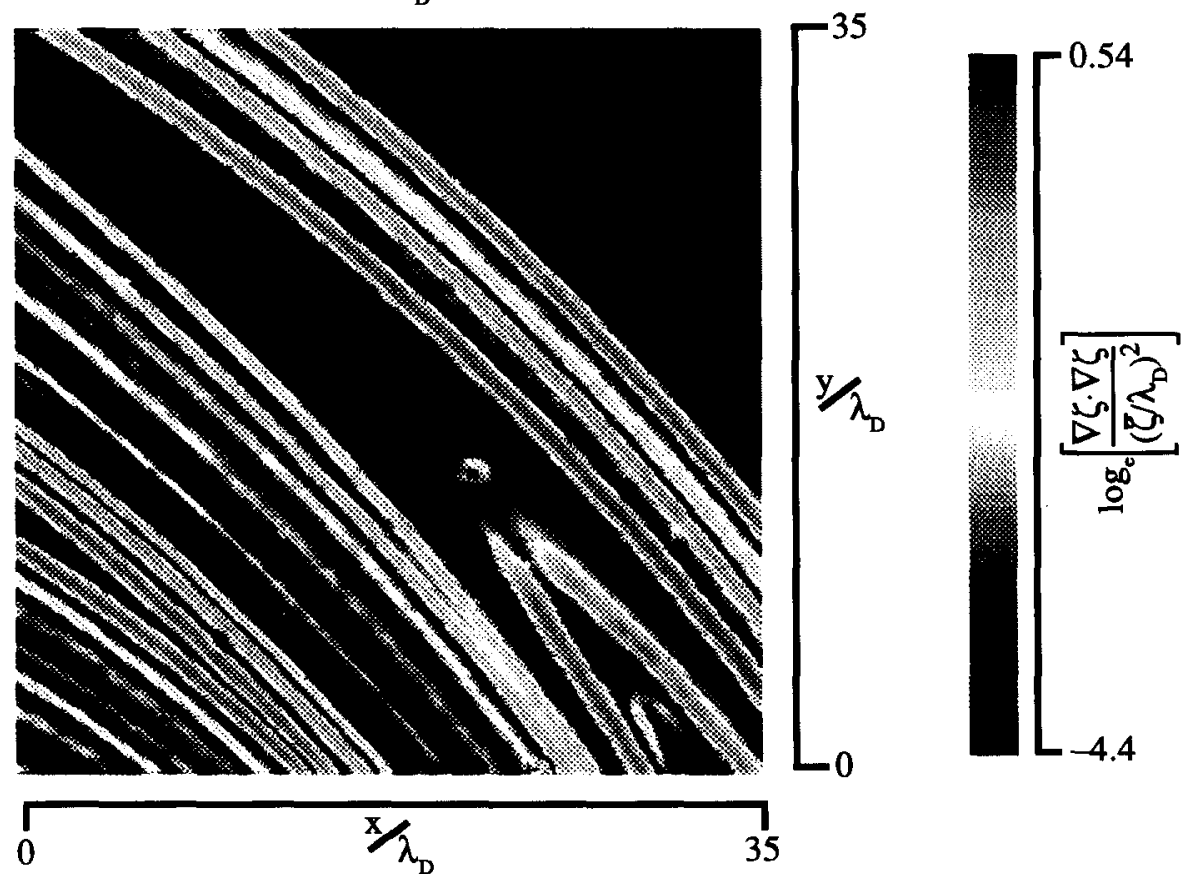

Fig. 4. The logarithm of the scalar energy dissipation rate field $\log _{e}[\nabla \zeta \cdot \nabla \zeta(\mathbf{x}, t)]$ for two different regions of the journal bearing flow of Fig. 2. (a) Example of a parallel region of the flow where the layers are essentially all aligned uniformly in the same direction. This is the resulting scalar dissipation field obtained from box 31 in Fig. 1. (b) Two-dimensional scalar dissipation field $\log _{e}[\nabla \zeta \cdot \nabla \zeta(\mathbf{x}, t)]$ calculated in box 7 , an example of an intermediate region or one in which the layers show some deviation from a strictly parallel formation. 

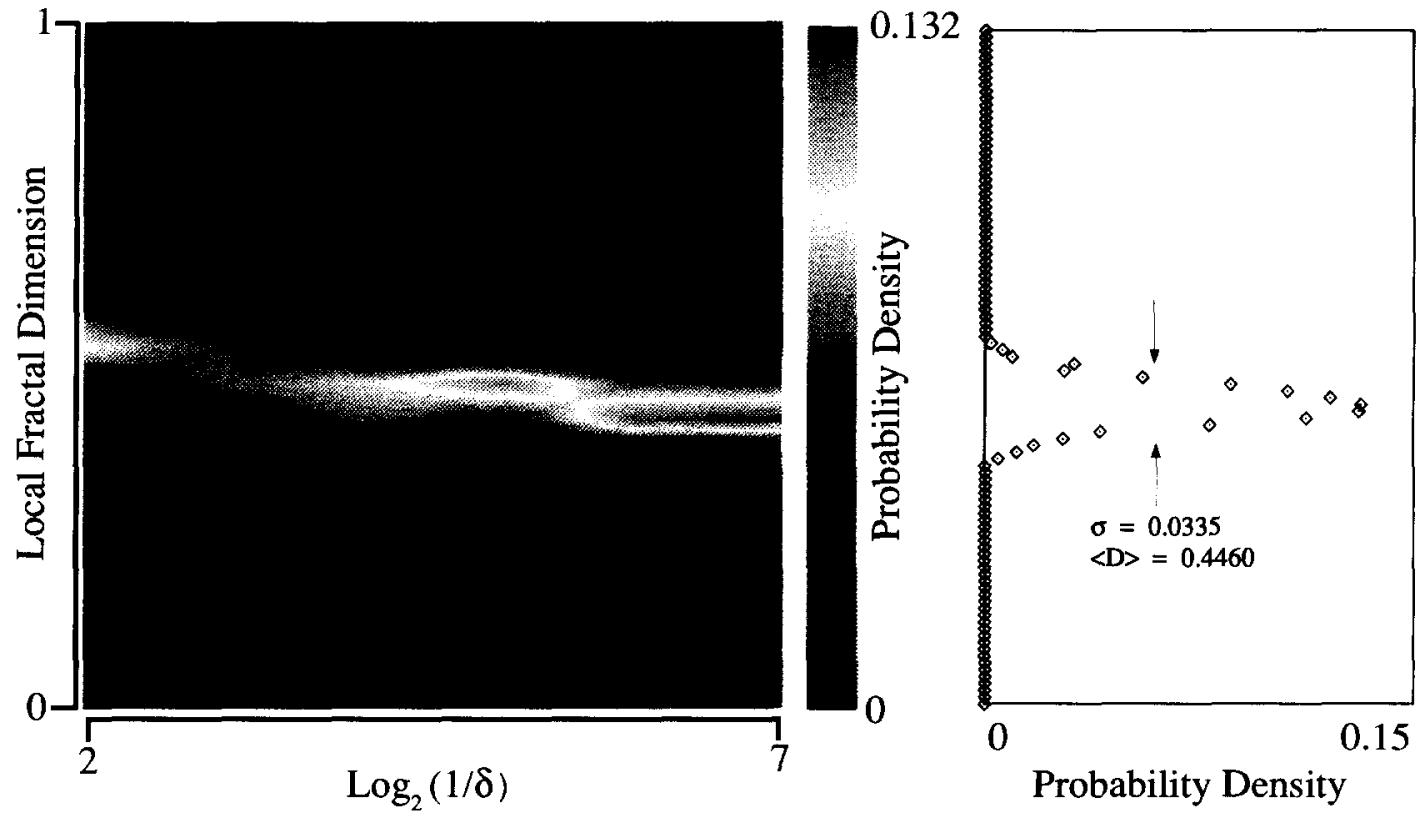

Fig. 18. The probability density plot of all of the local fractal dimension values from all 8000 test cases of digitized irregular cantor sets with dimension $=0.40$. A cumulative probability density curve for all of the local fractal dimension values in the range $4 \leqslant \log _{2}(1 / \delta) \leqslant 7$ is shown beside it. These test cases serve as a 'yardstick' with which to measure the fractal behavior of the experimental data.
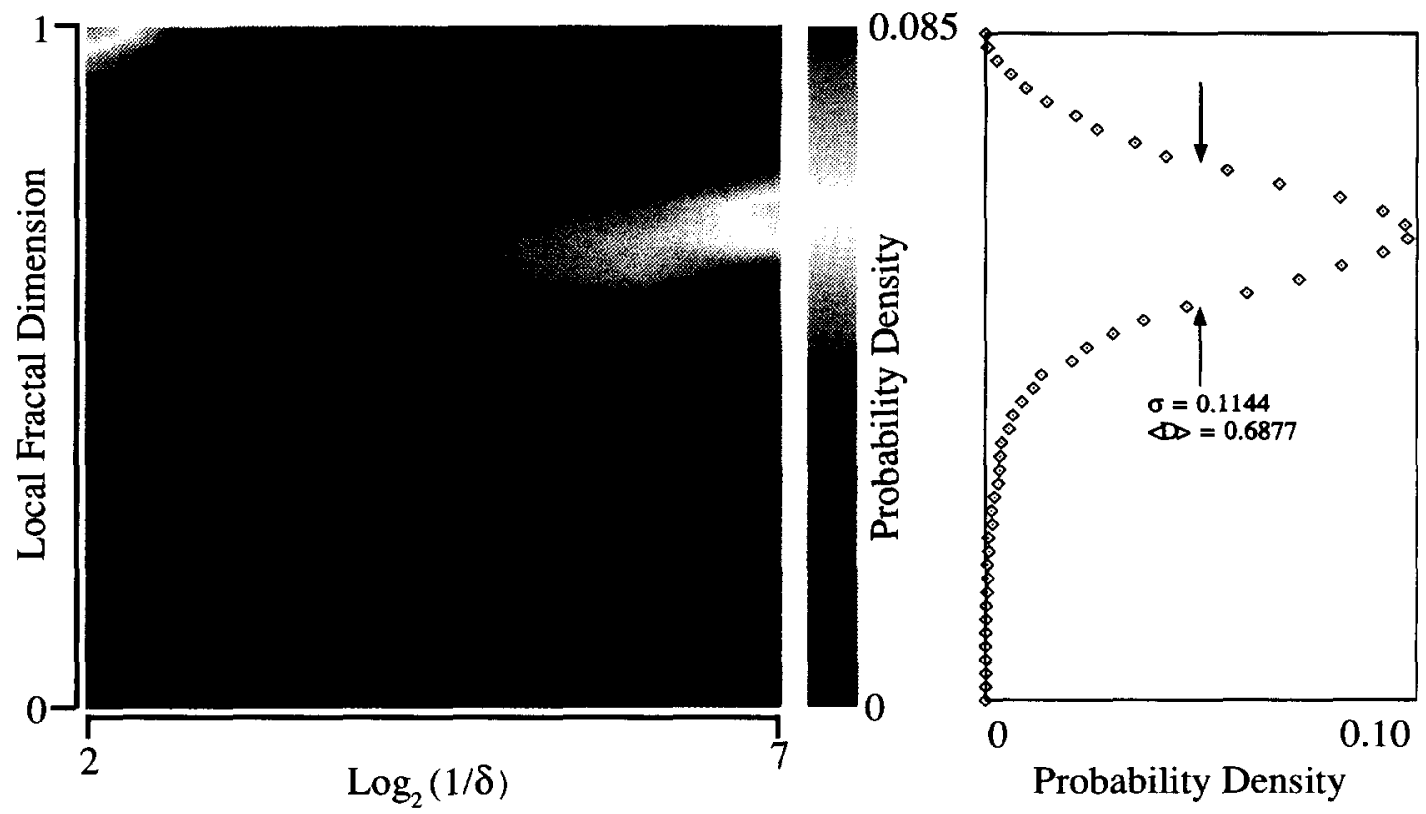

Fig. 22. The probability density plot of all of the local fractal dimension values from the chaotically advected data set. A cumulative probability density curve for all of the local fractal dimension values in the range $4 \leqslant \log _{2}(1 / \delta) \leqslant 7$ is shown beside it. From this plot it is obvious that the whole field is not described by one single fractal dimension. 

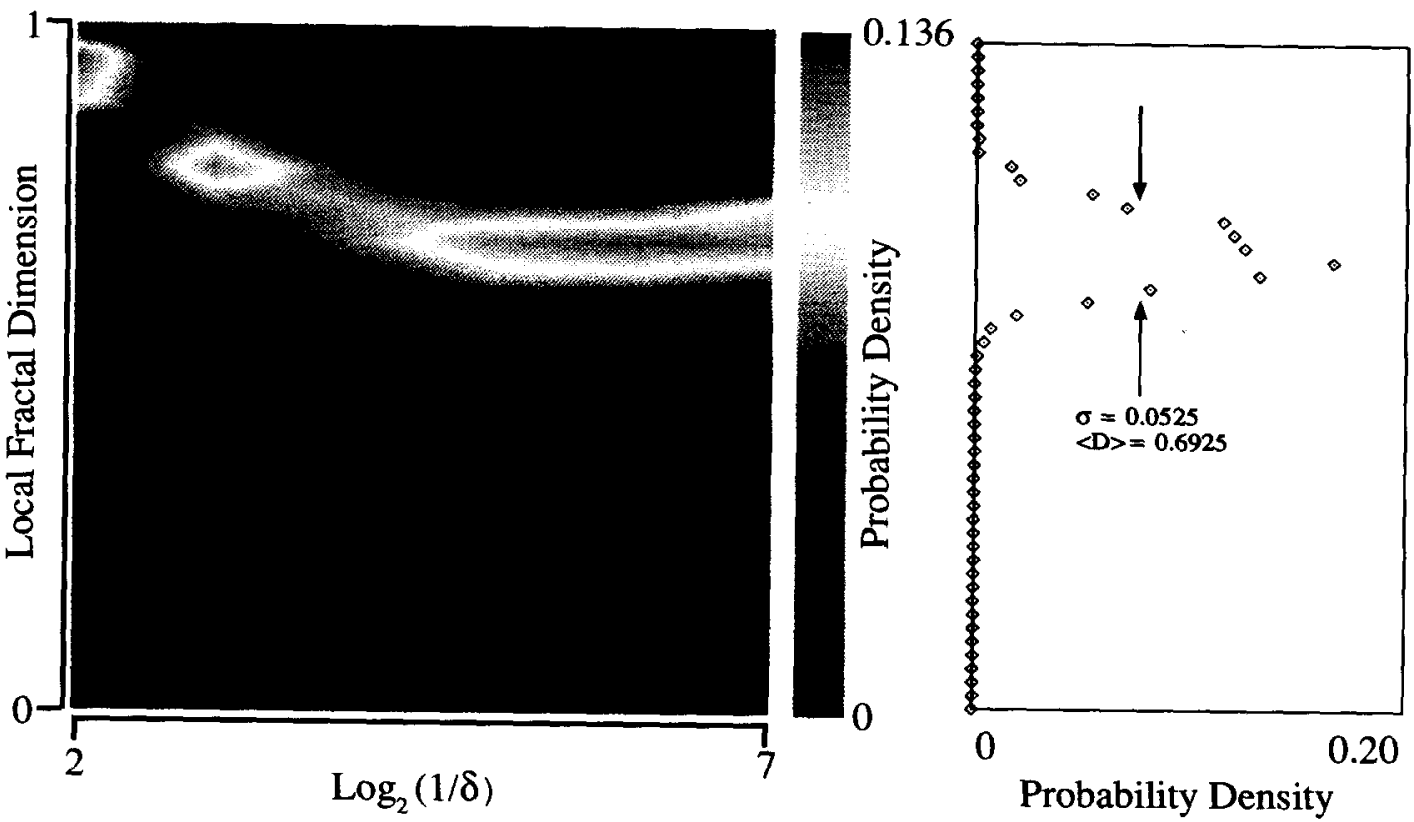

Fig. 23. The probability density plot of all of the local fractal dimension values from a parallel region of the chaotically-advected data set. A cumulative probability density curve for all of the local fractal dimension values in the range $4 \leqslant \log _{2}(1 / \delta) \leqslant 7$ is shown beside it. It shows nearly fractal behavior but the standard deviation in the local fractal dimension values is still approximately 2 times the value obtained from the test cases.
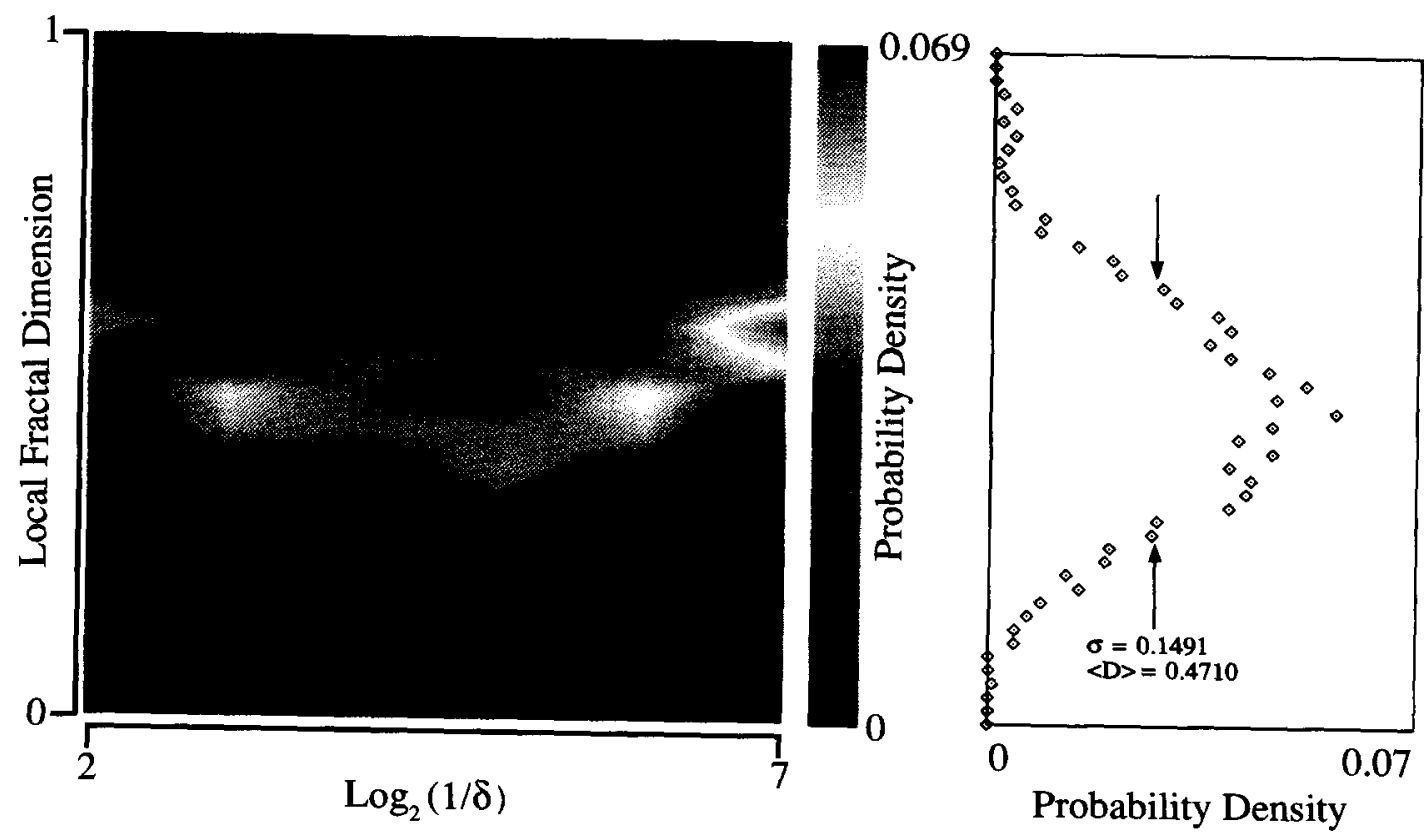

Fig. 24. The probability density plot of all of the local fractal dimension values from a non parallel region of the chaotically-advected data set. A cumulative probability density curve for all of the local fractal dimension values in the range $4 \leqslant \log _{2}(1 / \delta) \leqslant 7$ is shown beside it. This region shows absolutely no fractal behavior. 

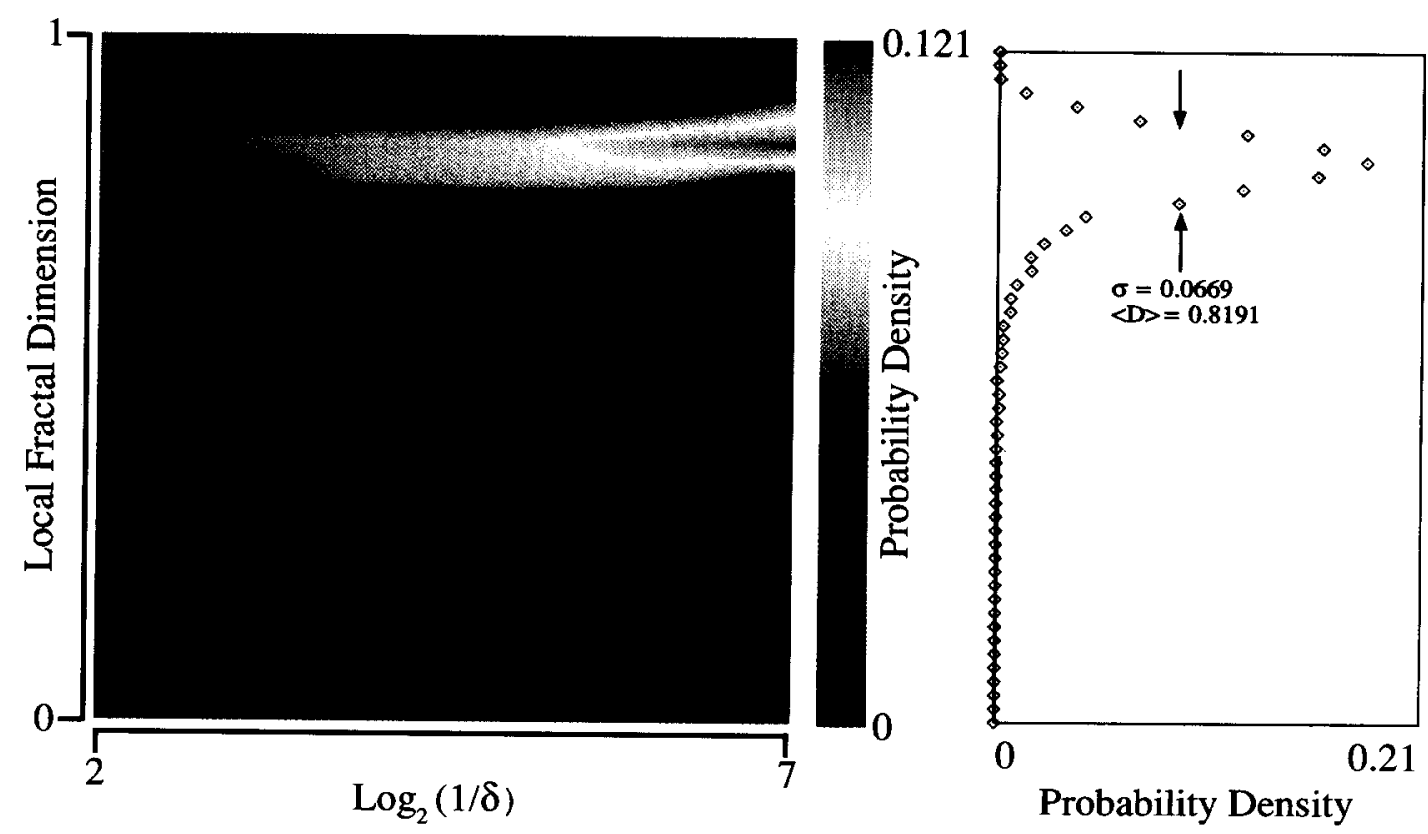

Fig. 27. The probability density of the local fractal dimension values from a typical region of the turbulent data set. Beside it is a cumulative probability density curve for all of the local fractal dimension values in the range $4 \leqslant \log _{2}(1 / \delta) \leqslant 7$. This region shows slight fractal behavior with a standard deviation in the local fractal dimensions that is approximately 3 times the value obtained from the test cases. 
Given the obvious qualitative similarities in the highly convoluted layered structures seen in such chaotic flows and in turbulent flows like that in Fig. 1, one might hope that the analytical tools that have been fruitfully applied to chaotic mixing might be applicable to turbulent mixing as well.

The widely held view is that turbulent flows are far more complex than these lower-dimensional and periodic chaotic flows, and that insights drawn from chaotic mixing might therefore have limited applicability to turbulent mixing. Indeed, when viewed on relatively large scales, the hydrodynamics of turbulent flows are undeniably more complex. However, at the small scales it becomes more difficult to argue for fundamental differences between these two types of flows. In particular, when turbulent mixing is viewed in a Lagrangian frame, the underlying hydrodynamics at the smallest velocity gradient length scales reduce to a locally uniform strain flow with time-varying principal axes, which leads to a continual stretching and reorientation of material elements in the flow. On slightly larger length scales, differential rotation rates resulting from vorticity gradients fold the drawn-out material elements back onto themselves in a manner reminiscent of the Smale horseshoe in the chaotic flow examples. The picture that results is one of turbulent mixing as a repeated stretching and folding of material elements by time-varying strain-rate and vorticity fields, much as in the chaotic flows, albeit in three dimensions rather than two, and without the simplicity of time-periodicity in the strain-rate eigenvector orientations. Moreover, unlike the closed chaotic mixing systems referred to above, a key feature of many turbulent flows is the continual entrainment of fluid into the mixing regionincreasingly more fluid participates in the Lagrangian mixing process as the flow evolves in time. In fact, it is precisely the large fluid entrainment rates that make turbulent flows attractive for most practical applications involving fluid mixing. Keeping in mind these differences, it is tempting to imagine that the underlying similarities in the mixing dynamics at the small scales in a turbulent flow and in a chaotic flow may lead to similar mixing patterns. The tools developed for describing and predicting the mixing properties of chaotic flows might then be productively applicable to turbulent flows as well. Such flows would be relatively simple at the smallest scales, and demonstrate the complexity traditionally associated with turbulence only in the aggregate. This view of turbulent mixing built up from the small scales naturally suggests certain universal scalings and Reynolds number asymptotics traditionally associated with fluid turbulence.

\subsection{Present work}

Within the context of the above discussion, the objective here is to compare various scaling properties of the mixing process at the small scales of high Reynolds number, three-dimensional, unsteady, open turbulent flows with those in low Reynolds number, two-dimensional, time-periodic, closed chaotic flows. To this end the experimental analyses are focused on two flows. We take the creeping flow between eccentric cylinders executing a discontinuous periodic rotation schedule as a canonical representation of the fundamental mixing patterns produced by the closed chaotic flow systems referred to above. The open turbulent flow in the self-similar far field of an axisymmetric jet at high Reynolds number is taken to represent the quasi-universal mixing properties at the small scales of turbulent flows. In each of these flows, measurements of the mixing of a dynamically passive, conserved scalar field $\zeta(\mathbf{x}, t)$ by the underlying flow field are used to analyze geometric scaling properties of the support set on which the associated scalar energy dissipation rate field $\nabla \zeta \cdot \nabla \zeta(\mathbf{x}, t)$ is concentrated.

For both flows we examine two measures of the dissipation support set geometry. The first closely resembles the 'striation thickness distribution' of Ottino [4]. In practice, the 
STD is difficult to measure accurately, especially in a system with significant diffusion. For this reason we instead work with the set of points forming the convoluted surface on which the local centers of the scalar dissipation layers reside. From this surface we determine the local one-sided surface-normal distance to the next adjacent dissipation layer. Repeating this over the entire surface produces the distribution of dissipation layer separation distances, which can be readily determined in practice even in highly diffusive systems. We then compare the distributions obtained in the chaotic and turbulent flows as a relatively sensitive measure of the geometry of the dissipation field at the small scales.

Second, we characterize the dissipation support geometry by examining the possible fractal scaling properties of the set of points within which the scalar dissipation rate exceeds a threshold level. Prior attempts at assessing the fractal properties of turbulent mixing have focused largely on the geometry of isoscalar surfaces, and have found that the results depend strongly on the chosen isoscalar value. In contrast, owing to the highly localized layer-like sheets on which the scalar dissipation field is concentrated (see Fig. 1), the geometric scaling properties of the dissipation field are comparatively insensitive to the choice of dissipation threshold. We assess the applicability of a fractal description of the dissipation support for the mixing process in both the chaotic and turbulent flows.

\section{EXPERIMENTS}

\subsection{Turbulent flow scalar fields}

Conserved scalar fields $\zeta(\mathbf{x}, t)$ as shown in Fig. 1 were measured in the self-similar far field of an axisymmetric turbulent jet at outer scale Reynolds numbers $\operatorname{Re}_{\delta} \equiv(\mathrm{u} \delta / v)$ as high as 6000 , and with resolution finer than the local strain-limited molecular diffusion scale $\lambda_{D}$. Since the imaged volume is small in comparison with the local outer flow scale $\delta$, and comparable to the inner scale $\lambda_{v}$ of the turbulence, the resulting small scale structure of the scalar field will be essentially independent of both the Reynolds number and the particular turbulent shear flow, and should be generic to large Schmidt number $S c \equiv(v / D)$ mixing in high Reynolds number turbulent flows. Details of the measurement technique can be found in ref. [1]. Briefly, we measure the aqueous concentration of a laser fluorescent dye carried by one of the fluids in a small three-dimensional spatial volume located 235 jet momentum diameters $(1.15 \mathrm{~m})$ downstream of the jet source and $13 \mathrm{~cm}$ off the jet centerline, well into the self-similar far field of the flow. The dye mixture fraction is a conserved scalar with $S c \approx 2075$. This is measured in time throughout the volume by stimulating laser-induced fluorescence from dye-containing fluid from a collimated laser beam and imaging this onto a high-speed, planar, photodetector array. The beam is swept in a raster fashion through the desired volume in the flow, and the resulting laser-induced fluorescence intensity is collected onto the $256 \times 256$ pixel imaging array, which can be driven at continuous rates in excess of 140 data planes per second. The fluorescence data from the array is serially acquired at 8-bits true digital depth, and continuously written in real time to a $3.1 \mathrm{~GB}$ high-speed parallel transfer disk rank capable of accommodating more than 50000 such $256^{2}$ spatial data planes. The spatial separation between adjacent points within each data plane, and between adjacent data planes within each data volume, is smaller than the local strain-limited molecular diffusion length scale $\lambda_{D}$ of the scalar field, where $\left(\lambda_{v} / \delta\right) \approx 11.2 \cdot \operatorname{Re}_{\delta}{ }^{-3 / 4}$ and $\lambda_{D} \approx \lambda_{v} \cdot S c^{-1 / 2}$. The scales $\lambda_{v}$ and $\lambda_{D}$ are respectively proportional to the classical Kolmogorov and Batchelor scales (which are defined solely on dimensional grounds) but are roughly a factor of 6 larger. Similarly, the temporal separation between adjacent data planes within each data volume and, depending on the number of spatial planes, between the same data plane in successive data volumes, is 
shorter than the local molecular diffusion scale advection time $\lambda_{D} / u$. This resolution, together with the high signal quality attained, allows accurate differentiation of the measured conserved scalar data in all three space dimensions and in time to determine the components of the true local instantaneous scalar gradient vector field $\nabla \zeta(x, t)$ at every point in the data space.

Figure 3 shows the scalar dissipation rate data in two typical spatial data planes. Note all quantities are normalized by the inner scales $\lambda_{D}$ and $\langle\zeta\rangle$ of the underlying turbulent flow. Each $\dot{\nabla} \zeta \cdot \nabla \zeta(\mathbf{x}, t)$ plane is obtained by direct linear central differencing of the scalar field data in the three adjacent scalar planes; no explicit smoothing or filtering is used in evaluating the three components of the scalar gradient vector field $\nabla \xi(\mathbf{x}, t)$. Owing to the wide range of dissipation rates (see Fig. 1(b)), we show $\log _{e} \nabla \zeta \cdot \nabla \zeta(\mathbf{x}, t)$ in Fig. 3 to allow the structure at low dissipation rates to be readily seen. Notice that essentially all of the molecular mixing occurs in thin sheet-like scalar dissipation layers.

\subsection{Chaotic flow scalar fields}

Measurements of the scalar field $\xi(\mathbf{x}, t)$ in chaotic mixing are obtained by digitizing photonegatives provided by J. M. Ottino of his experiments on the flow between eccentric rotating cylinders - the 'journal bearing flow'. Details of the experimental apparatus and flow can be found in ref. [12]. Briefly, two cylinders of radii $R_{1}$ and $R_{2}$, with centers displaced by $\varepsilon$, are respectively rotated in a discontinuous time-periodic fashion through angular displacements $\vartheta_{1}$ and $\vartheta_{2}$ during each period. At Reynolds numbers sufficiently low for the creeping flow approximation, the annular flow field is determined by the two geometric parameters $\left(R_{1} / R_{2}\right)$ and $\left(\varepsilon / R_{2}\right)$, held fixed at $1 / 3$ and $3 / 10$ respectively, and the two displacement angles, which can be expressed as $\Omega \equiv\left(\vartheta_{1} / \vartheta_{2}\right)$ and $\vartheta \equiv \vartheta_{2}$. For the case considered here, $\Omega=-3$, and $\vartheta=4 \pi$. The fluid in the annulus is glycerine, a region of which is initially marked with fluorescent dye. Owing to the low diffusivity of the dye in glycerine, the Peclét number $(R e S c)$ is large even though $R e \rightarrow 0$. The flow is allowed to mix this tracer for a given number of rotation periods, after which the dye pattern in the annulus is photographed. The scalar field in the resulting $3 \mathrm{in.} \times 5 \mathrm{in}$. photonegatives is digitized on a light table using the same imaging array described above. The negative is divided into individual windows, as indicated in Fig. 2, and each window digitized to $256^{2}$ resolution and 8-bits signal depth. The repeated stretching and folding of the dyed fluid by strain and differential rotation can be seen to lead to a highly layered (lamellar) structure in the scalar field $\zeta(\mathbf{x}, t)$. The basic features of this field are established after just a few periods; further iterations principally increase the fine structure in an apparently selfsimilar fashion. Within each measurement window, the inherently two-dimensional scalar energy-dissipation rate field $\nabla \zeta \cdot \nabla \zeta(\mathbf{x}, t)$ is computed using linear central differences on this discretized field. Two typical examples of the resulting scalar dissipation fields are shown in Fig. 4.

There are difficulties inherent in this approach that may influence the results. These include errors in the photonegatives themselves, as discussed in ref. [12], among which parallax effects and the nonlinear film response are likely to be the most prominent. Other nonidealities are introduced by our processing of these negatives, e.g. minor flaws in the negative quality occasionally produce spots in the dissipation field. These imperfections appear to be sufficiently infrequent as not to significantly affect the results obtained. A potentially more serious limitation stems from the restricted spatial resolution achievable by indirect imaging measurements from photonegatives. With increasing number of periods, the 'striations' become thinner and eventually cannot be accurately resolved, limiting the maximum period $n$ for which accurate measurements can be obtained. Results 
of Muzzio et al. [13] suggest that, for relatively low values of $\Omega$ and $\vartheta$, full self-similarity in the distribution of stretching values may not be achieved until $n \approx 10$. The extent of similarity, however, appears likely to depend on the total stretching that has occurred, while at the same time the striation thicknesses that need to be resolved decrease as the total stretching increases. The central issue, then, is whether or not the striations can still be adequately resolved at a stage in the development of the flow for which self-similarity is reached. Comparing Fig. 1(b) of ref. [13] ( $n=10$ for $\Omega=3$ and $\vartheta=2 \pi)$ with our Fig. 2 suggests a roughly similar total stretching, while their Fig. 4 indicates that this stage of development is indeed marginally sufficient for self-similarity.

\section{DISSIPATION LAYER SEPARATIONS}

The detailed structure of the mixing patterns that result from the repeated stretching, reorientation, and folding of material elements in the chaotic journal-bearing flow have been discussed in terms of the striation thickness distribution (STD) by Ottino [8,9]. The STD is, however, notoriously difficult to measure, in part for the reasons outlined above. Consequently, numerical simulations of mixing in chaotic flows (e.g. refs [13-15]) have focused instead on the distribution of stretching values (DSV), which is closely related to the STD and can be readily obtained if access to the underlying velocity field is available. For two-dimensional, time-periodic, creeping flows of the type classically used in studies of chaotic mixing, the velocity field is known, and the numerical studies referred to above have used this to demonstrate remarkable self-similarity and scaling properties of the DSV. In turbulent flows, however, access to the fully three-dimensional, time-varying velocity field is only recently beginning to become available [2], and thus routine access to the DSV is not yet possible. A comparison of the mixing patterns in chaotic and turbulent flows must, therefore, return to measures of the spatial structure of the scalar field. Owing to the inherently sheet-like structure of the scalar dissipation field, we will compare the distribution of dissipation layer separations. Once the effects of initial conditions have become relatively unimportant, this layer separation distribution will be closely related to both the STD and the DSV. Unlike the STD, however, the compact support of the scalar dissipation field allows layer separation distributions to be somewhat more easily measured and, unlike the DSV, this can be obtained in both the chaotic and turbulent flows. The procedure we use for determining these distributions is described in Section 3.1; results for the chaotic and turbulent flows follow in Sections 3.2 and 3.3.

\subsection{Algorithms}

Determining the dissipation layer separation distribution first requires that the set of points containing the dissipation layer centers must be found within each two-dimensional scalar dissipation plane from the chaotic flow experiments, as shown in Fig. 4, and each three-dimensional scalar dissipation volume from the turbulent flow experiments as shown in Figs 1 and 3. We use an algorithm for automatically finding the dissipation layer centers; however, as is often the case in 'machine vision' applications, this is more difficult to do well than it might appear. Extensive tests conducted with various algorithms revealed features necessary for reliably identifying the layer centers in the algorithm that was finally used. This algorithm is based principally on the scalar gradient vector field $\nabla \zeta(\mathbf{x}, t)$. Briefly, it begins by thresholding the gradient magnitude field (dissipation rate field) at some chosen level. Next, it identifies the edges of the regions in which the dissipation rate is above the threshold value. To do this, for each point the local unit normal vector for the 
(a)

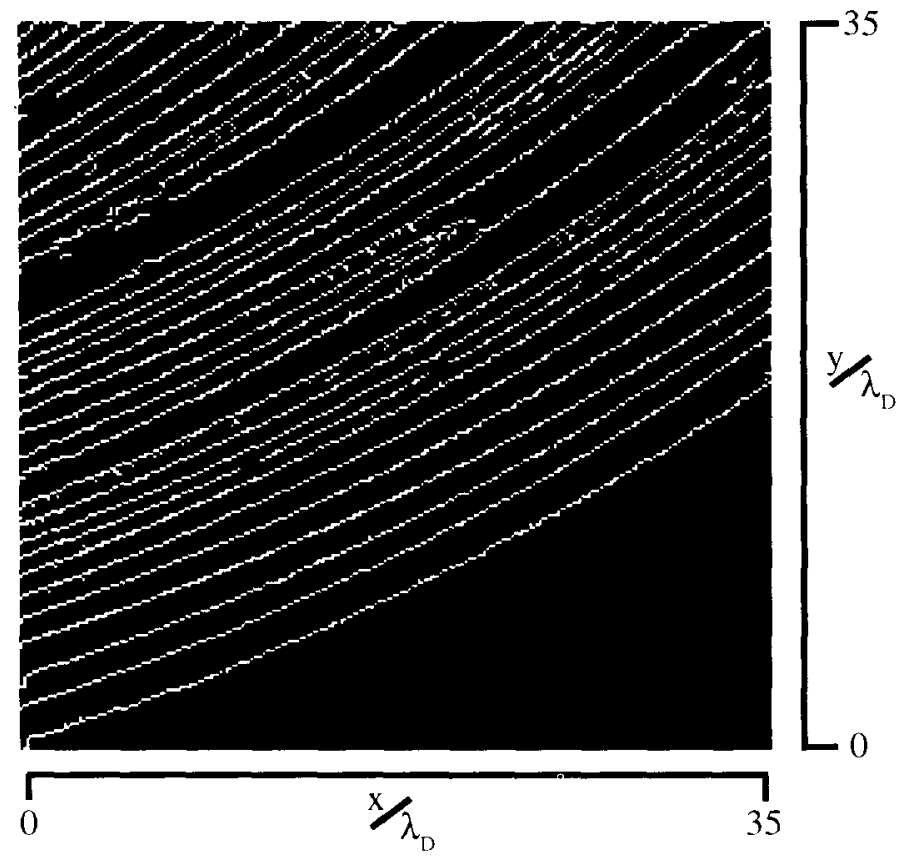

(b)

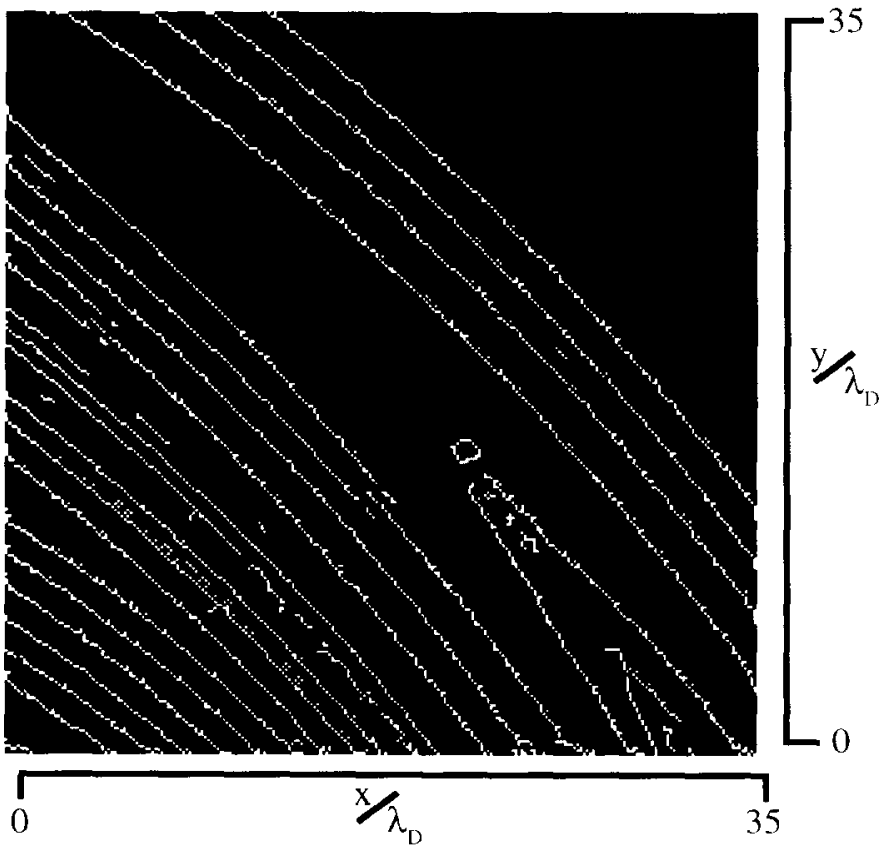

Fig. 5. Fields representing the collection of points used to describe the location of the centers of the scalar energy dissipation layers in the chaotic flow of Fig. 2. Each point reflects a local maximum in the scalar energy dissipation rate fields of Fig. 4 where once again (a) represents an example of a parallel flow region and (b) represents an intermediate region. Note the cleanliness of the layer definitions resulting from the application of the center finding algorithm to this two-dimensional flow. Layer separation PDFs are obtained by measuring the distances between adjacent layers at each point defining the layers. 
(a)

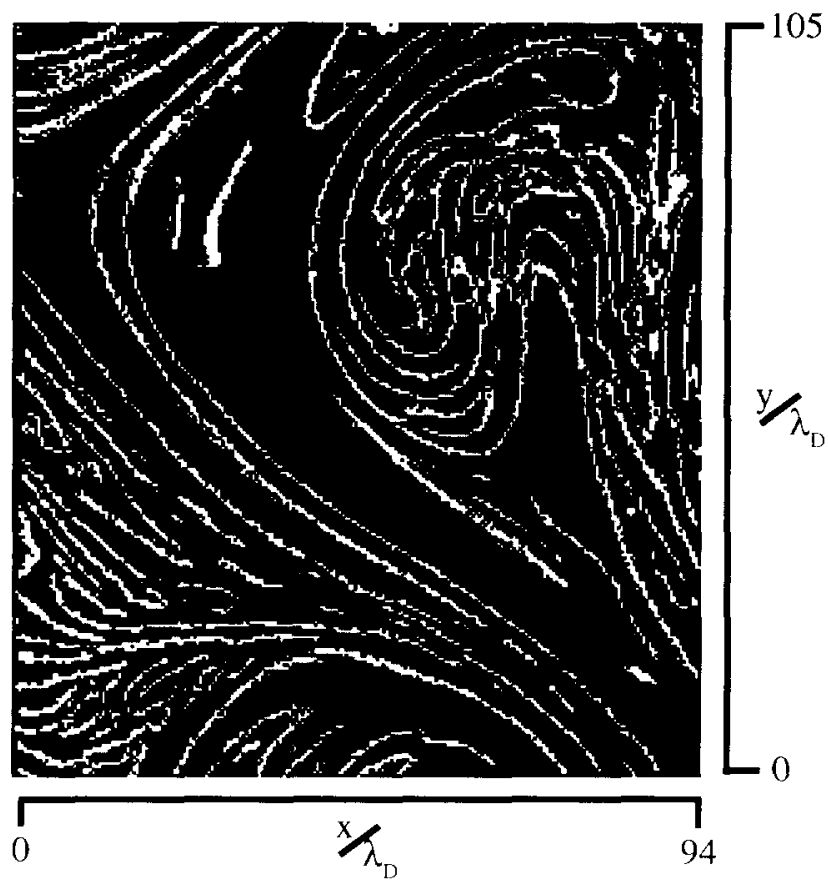

(b)

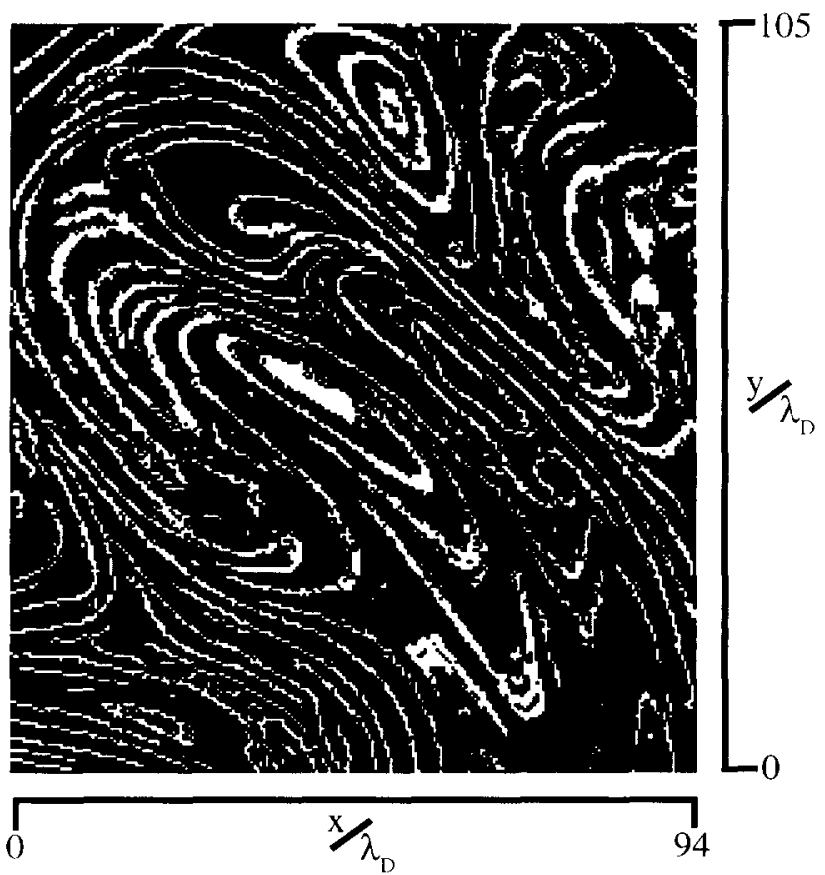

Fig. 6. Fields representing two-dimensional cuts of the collection of points used to describe the location of the centers of the scalar energy dissipation layers in the fully three-dimensional turbulent shear flow of which Fig. 1 is an example. Each point reflects a local maximum in the scalar energy dissipation rate fields of Fig. 3 (a) and (b). Note that the intersections of the three-dimensional surface defining the center of a layer with the two-dimensional cut plane allows adjacent pixels within the plane to be identified with the surface wherever it passes through the plane at more of a grazing incidence. A thick layer will appear wherever the sheet normal direction is pointing essentially perpendicular to the cut plane. 
layer is determined based on the local gradient vector information. The edges of the layer are then found by marching in both directions along this unit normal direction until one of two criteria are met: (i) the scalar dissipation at the candidate edge point drops below the chosen threshold level, or (ii) the dot product between the scalar gradient unit vector at the original point and that at the candidate edge point changes sign. The resulting edge locations for the dissipation layers are then used to identify candidate midpoints representing the dissipation layer centers. A further check is made among these candidate dissipation layer center points to insure single pixel thickness for the resulting surface before finally admitting a pixel to the set of layer center points.

Figure 5 shows the resulting dissipation layer centers identified by this algorithm for the two data planes in Fig. 4. Note that most of the layer centers are accurately determined, though there are instances where very weak dissipation layers (the color scale in Fig. 4 is logarithmic) produce either 'holes' or 'erroneous layers'. Generally speaking, algorithms capable of reliably detecting very weak layers produce many erroneous layers. Conversely, attempts to reduce the number of erroneous layers tend to miss the weaker dissipation layers. The final algorithm used is a compromise between these two competing interests, representing roughly an 'optimum' among the various identification strategies attempted. Obvious means for further reducing the number of small spurious layers in the chaotic flow in Fig. 5 tend to remove valid layers in the turbulent flow, owing to the more convoluted nature of the dissipation field in Fig. 3. Examples of the dissipation layer centers for these two planes are shown in Fig. 6, which show that most of the layer centers are accurately identified. The more highly folded character of the dissipation layers in the turbulent flow, together with the three-dimensional nature of the data, make identification of individual layers even conceptually more difficult than for the nearly parallel layers in the twodimensional chaotic flow data. Notice also that, unlike the inherently two-dimensional surface of layer centers in the chaotic flow, intersections of the three-dimensional surface resulting from the turbulent flow data with any two-dimensional plane, as in Fig. 6, allows adjacent pixels within the plane to be identified with the surface wherever it passes through the plane at a relatively shallow angle.

The influence of imperfections that survive the layer center detection algorithm is considerably reduced by the algorithm used to determine the layer separation distances. This layer separation algorithm begins with the surface of dissipation layer centers, and aims to compute the surface-normal distance in either two or three dimensions from any point on the surface to the next neighboring part of the surface, in a manner that is relatively insensitive to small holes or spurious layers. At each point on the surface, the local surface-normal axis is determined from the scalar gradient vector orientation at neighboring points. The algorithm marches along this axis, staying on one side of the surface (to avoid double-counting), until the next intersection with the surface. The search direction along this axis is accordingly determined by which of the four quadrants (2D) or eight octants (3D) $\nabla \zeta(\mathbf{x}, t)$ points in. To reduce the effects of imperfections in the surface, the search for the next intersection is conducted within a $15^{\circ}$ half-angle wedge (2D) or cone (3D) centered on the local surface-normal axis.

Tests conducted with synthetically generated surfaces having varying levels of imperfection verify that the resulting distribution of layer separations can be accurately obtained. An example is shown in Fig. 7(a). In this case, a synthetic scalar field was specified as $\zeta(\mathbf{x})=\sin \left(x^{2}\right)$ over $0<x<3 \pi$. From the associated scalar dissipation field, the resulting ideal cumulative distribution of layer separations can be obtained analytically. This agrees well with the distribution obtained from the procedure described above, as shown in Fig. 8. (Since the probability density for this discrete test case is not continuous, we instead compare the cumulative distributions. For the continuous separation distances in the 

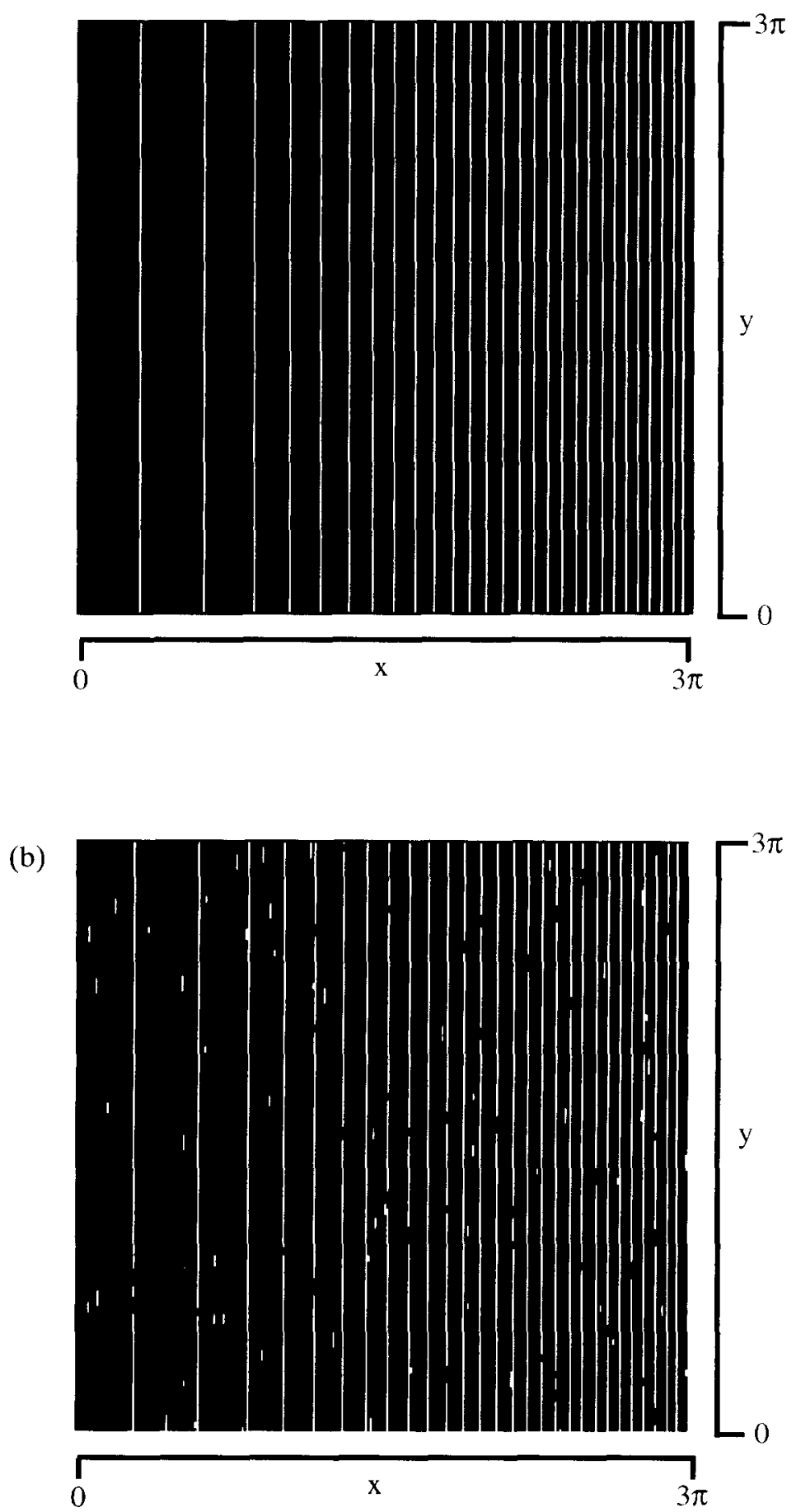

Fig. 7. Layer center definition fields for a test case that was formulated in terms of a conserved scalar field $\zeta(\mathbf{x}, t)$ that varied as $\sin \left(x^{2}\right)$. The scalar energy dissipation field in this case varies as $\nabla \zeta \cdot \nabla \zeta(\mathbf{x}, t)=4 x^{2} \cos \left(x^{2}\right)$ so that the magnitude as well as the frequency of the layers vary with the coordinate $x$. Shown in (a) is the clean version of this field resulting from our layer maximum finding algorithm and in (b) is the field in (a) made noisy by the addition of intentional holes and extraneous layers in a random manner. Both were subjected to a comparison measurement of their individual layer separation distance cumulative distribution functions which can be seen in Fig. 8 . 
chaotic and turbulent flow cases, we will compare the derivative of the cumulative distributions, namely the probability densities.) In Fig. 7(b), a random pattern of holes and extraneous layers, of various sizes and locations, were added to mimic similar effects anticipated in the measured data. The imperfections in this case are meant to qualitatively match those present in the layer centers in Figs. 5 and 6. The layer separation distribution resulting from this imperfect layer center field is also shown in Fig. 8. Except for very small separations, the resulting distribution still follows the ideal distribution reasonably well, and continuous derivatives (probability densities) of both sets of data are similar for all but the smallest layer separations.

\subsection{Chaotic flow results}

The probability density of dissipation layer separations is computed separately for each of the 31 windows identified in Fig. 2 via the above procedure. The layer separation distribution resulting from ensemble statistics over the entire set of windows covering the flow is shown in Fig. 9. This result is roughly analogous to the distribution of stretching values $F_{n}(\lambda)$ given in Fig. 3 of ref. [13], where a power law scaling can also be identified in the logarithmic axes, and where evidence of self-similarity can be seen. However, large stretching values in their case correspond to small layer separations in our results and, for a given range of layer separations, the corresponding range of stretching values is much wider. While Fig. 9 gives the statistics for the entire ensemble of windows in Fig. 2, the distributions of layer separations in individual windows turn out to be very different. For this reason, it proves useful to broadly classify the dissipation field structure in various regions of Fig. 2 into three different types. The first of these are termed 'parallel' regions, referring to windows in which the dissipation layers are all essentially straight and very nearly parallel to one another. The second are termed 'nonparallel' regions, where the layers are not straight or generally not parallel to each other. Finally, windows whose overall dissipation layer structure contains features of both are termed 'intermediate'.

In Fig. 10 we show the distribution of layer separations for all the parallel regions only.

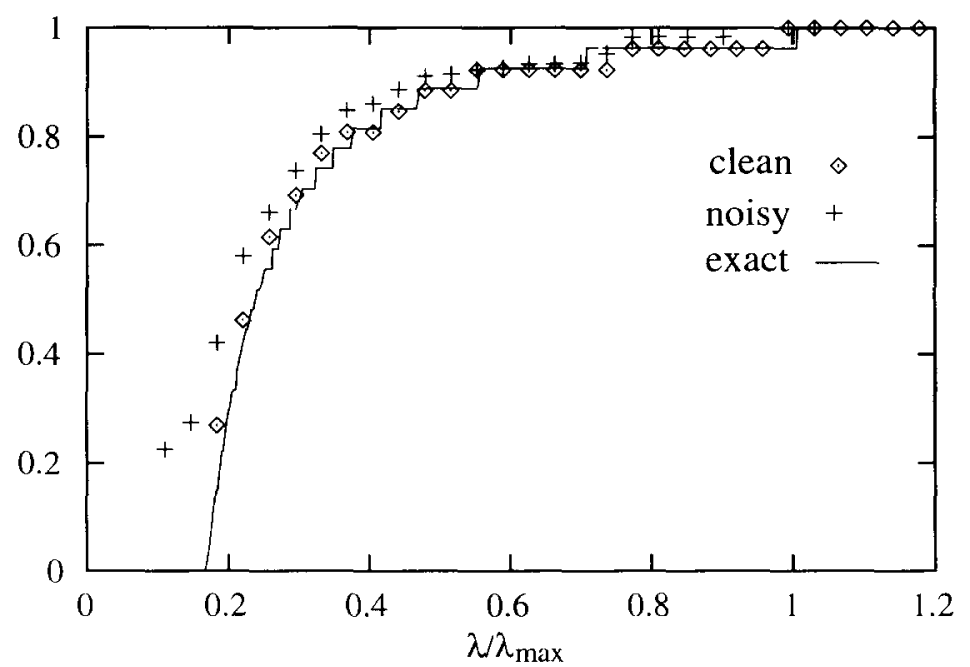

Fig. 8. Layer separation distance cumulative distribution functions for the test cases in Fig. 7. Shown as a solid line is the exact result for the cumulative distribution function for layers defined by $\nabla \zeta \cdot \nabla \zeta(x, t)=4 x^{2} \cos \left(x^{2}\right)$ over the range from 0 to $3 \pi$ obtained analytically. Overlaid with good agreement are the results obtained from our nearest-neighbor distance-finding algorithm for both the clean and noisy fields of Fig. 7 . 


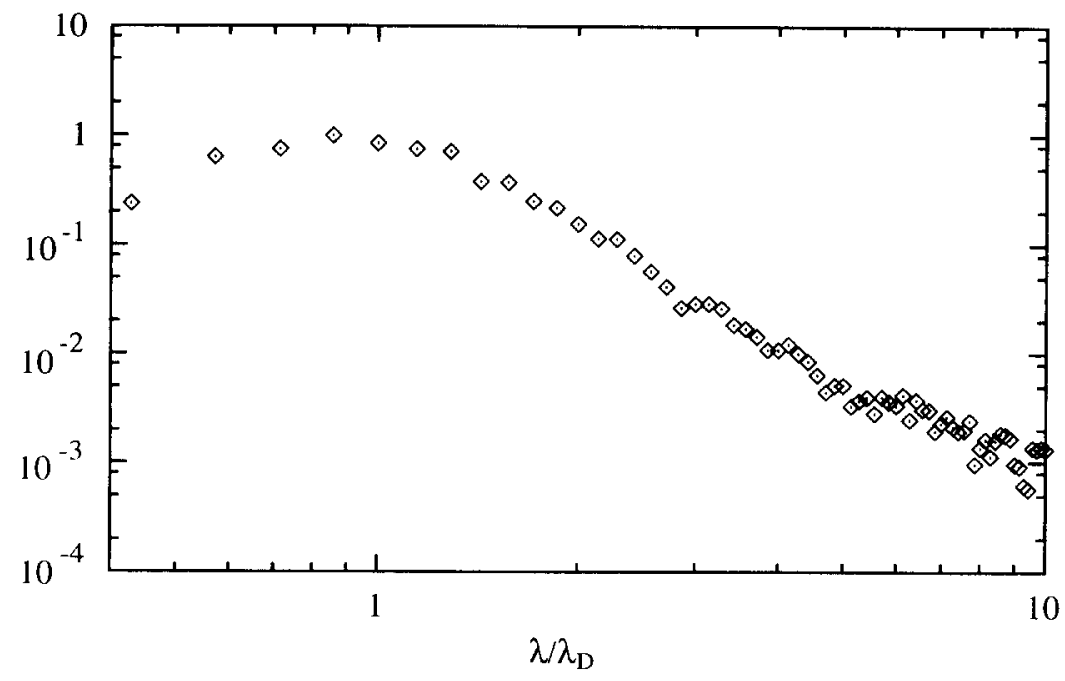

Fig. 9. Composite layer separation PDF for the chaotic flow between two eccentric cylinders as shown in Fig. 2. Result is formulated from the nearest neighbor calculation performed on the scalar energy dissipation rate field for all regions mapped out by the boxes in Fig. 2 regardless of the predominant layer arrangement within the box.

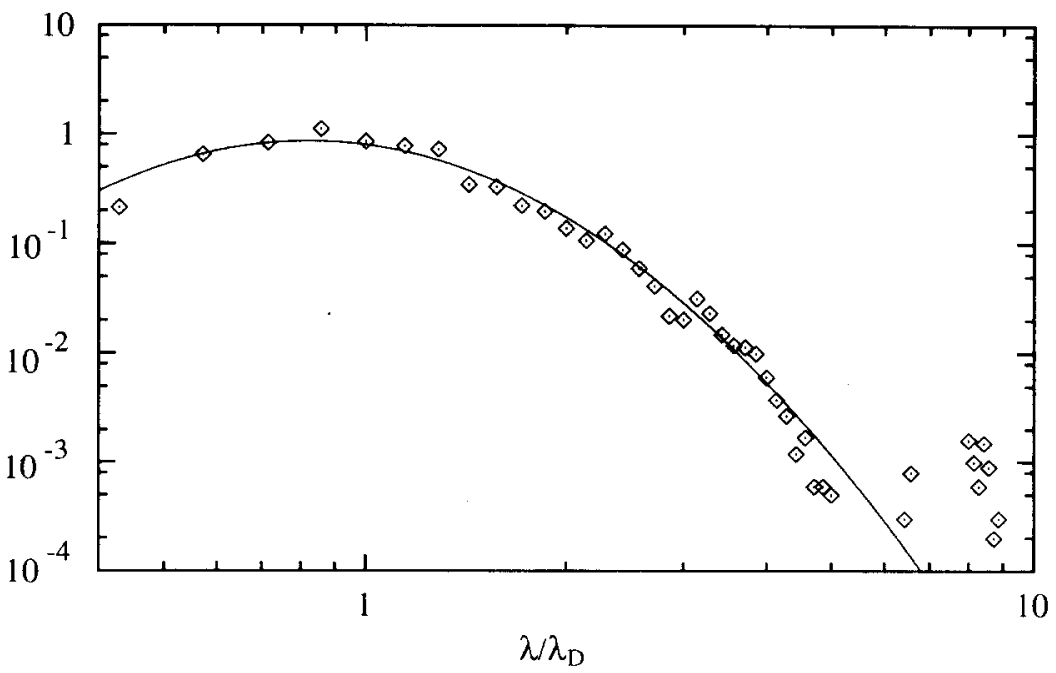

Fig. 10. Layer separation PDF for the parallel layer regions of the chaotic flow displayed in Fig. 2. Here the nearest-neighbor calculation was restricted to those regions of the flow, divided by the boxes in Fig. 2, comprising predominantly parallel arrangements to the scalar dissipation layers. Shown for comparison is a lognormal curve fitted with the same first and second moments of the resulting PDF.

The result can be seen to be distinctly different from that obtained over the entire flow field in Fig. 9, especially for large layer separations. Also shown in this figure is a lognormal distribution having the same first two moments, which can be seen to provide a good fit for all except the very largest layer separations. Such a lognormal form is expected from classical central limit arguments for multiplicative processes (e.g. $[13,14,16])$ provided that the number of independent multiplicative steps producing the layer separations is sufficiently large. One way to meet this provision is to require the flow to have undergone a large number of periods. Muzzio et al. $[13,14]$ show that, when collecting 
unconditional statistics over the entire flow, the highest stretching values (smallest layer separations) show lognormal scaling even after just a few repetitions (see their Fig. 3(a)). In a flow that has nonchaotic 'islands', however, departures from complete lognormality remain apparent in their DSVs at the lowest stretching values (largest layer separations), regardless of how many repetitions the flow has undergone. These departures from lognormal scaling can be attributed to locally low stretching values that lead to the persistence of large voids in the dissipation support. By collecting unconditional statistics, the continuing influence of these voids will be seen in both distributions of stretching values and layer separations. Following this reasoning, we might expect the lognormal scaling to hold at the smallest layer separations even after just a few periods. Indeed, by collecting conditional statistics as in Fig. 10, and thereby presumably avoiding areas of the flow strongly influenced by the voids, lognormal scaling can be seen over essentially the entire range of layer separations. The 'nonparallel' regions of the scalar field would then presumably be those most strongly influenced by the low strain rates in the vicinity of the voids, where the low stretching values lead to a slower reduction of the dissipation layer separations and a slower reorientation of the dissipation layers by the principal strain axes.

To test this, the individual distributions resulting for the parallel (no voids), nonparallel (voids), and intermediate regions are shown in Fig. 11. These reveal two distinct trends. First, in all three cases, the smallest separations follow a nearly lognormal distribution. For the parallel regions, this lognormality is preserved for all but the largest separations (up to $\lambda / \lambda_{D} \approx 6$ ). For the intermediate regions, departures from the lognormal distribution begin at $\lambda / \lambda_{D} \approx 4$, while in the nonparallel regions the lognormal form holds only up to $\lambda / \lambda_{D} \approx 3$. Second, as would be expected, large layer separations are rare in the parallel regions, and relatively prominent in the nonparallel regions. The closed nature of this flow suggests precisely these features. Since the total area of the surface of dissipation layer centers initially increases with time as the fluid is stretched and folded, the finite volume occupied by the flow requires a continual reduction in the average layer separation distance. With

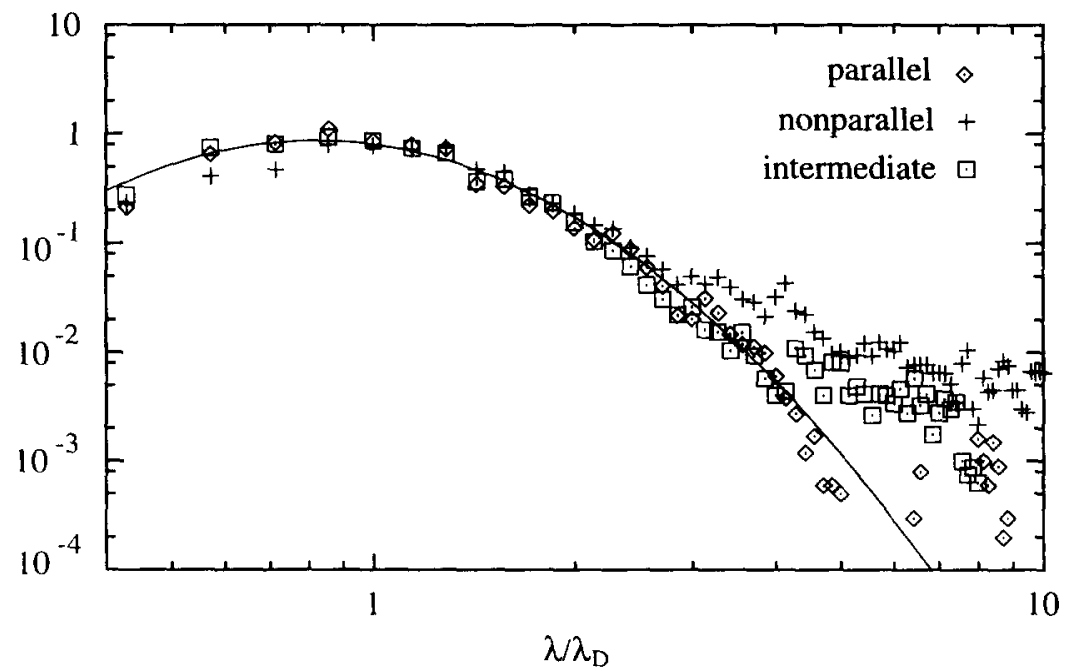

Fig. 11. Layer separation PDFs for the three distinct regions of the chaotic, two-dimensional, time-periodic, eccentric cylinder flow of Fig. 2 defined as parallel, nonparallel, and intermediate. Shown for comparison is a lognormal curve fitted with the same first and second moments of the PDF of layer separations for the parallel regions. Note that the deviation from this lognormal curve occurs at decreasing values of the distance separating neighboring layers as we move from the PDF for the parallel regions through the PDF for the intermediate regions to the PDF for the nonparallel regions. 
increasing time, large separations must become increasingly rare (though only at a slow rate set by processes near the voids) and smaller separations must become increasingly dominant. In each type of region, the smallest layer separations presumably result from areas in the flow that have experienced the largest cumulative stretching, while the largest layer separations correspond to the lowest total strain. As the number of periods increases $(n \rightarrow \infty)$, the distribution will tend toward lognormality. These distinctly different features near and away from voids are evident in our relatively early time results. Here, the parallel regions show strong evidence of lognormality, and are reminiscent of a later time when the space-filling tendency of the stretching and folding process require the existence of many more parallel layers than nonparallel ones. The fraction of the volume occupied by regions containing nearly parallel layers will then increase with the number of periods, due to this stretching and folding. The distribution for the whole flow field after a large number of periods will asymptotically approach that of just the parallel regions of the flow at the earlier times.

The persistent $(n \rightarrow \infty)$ influence of voids on mixing in chaotic flows with islands has been recognized previously for distributions of stretching values and Lyapunov exponents (e.g. $[17,18])$. The influence of initial voids has a similar effect in the distribution of dissipation layer separations. However, stretching values and Lyapunov exponents associated with the mixing process are not readily accessible in turbulent flows, while the dissipation layer separation distribution can be determined in a turbulent flow following precisely the same procedure as was done here for a chaotic flow. In the following section, we present results obtained from our turbulent flow measurements, and compare these with the results in Figs 9-11.

\subsection{Turbulent flow results}

Probability densities of dissipation layer separations were computed for each of 29 individual three-dimensional $256^{3}$ spatial data volumes of the type shown in Fig. 1 from our turbulent flow measurements at $R e_{\delta} \approx 3700$. These volumes were equally spaced in time, and spanned slightly more than 2.5 outer scale turnover times $(\delta / u)$ of the flow. The size of each volume was roughly $\left(2 \lambda_{v}\right)^{3}$, where $\left(\lambda_{v} / \delta\right) \approx 11.2 \cdot \operatorname{Re}_{\delta}{ }^{-3 / 4}$ is the local strain limited velocity gradient length scale in the turbulent flow, and $\operatorname{Re}_{\delta} \equiv(u \delta / v)$ is the local outer scale Reynolds number based on the length and velocity scales $\delta$ and $u$ characterizing the local mean shear in the flow. The scalar field within any volume thus represents the signature of the mixing process at the small scales of the flow. The dissipation layer separation distribution resulting from ensemble statistics over the entire set of spatial data volumes is shown in Fig. 12. A roughly -3 power law scaling can be seen in the distribution of layer separations over this range of length scales, reminiscent of the power law scale similarities typically found in high Reynolds number turbulent flows.

Although the ensemble-averaged distribution exhibits this -3 power law scaling, the individual dissipation layer separation distributions vary considerably from one data volume to the next, as shown in Fig. 13. The -3 scaling appears to result only from averaging over many such individual uncorrelated volumes, but is not evident in any single volume. However, while the distribution varies significantly at large layer separations, for small separations the results for all volumes are nearly the same. Though perhaps only coincidentally, there are interesting similarities evident when Fig. 13 is compared with Fig. 11 from the chaotic flow results. In both cases, the distributions for small separations are essentially invariant and show a roughly lognormal scaling, while for large separations the distributions are very different. In the chaotic flow, the multiplicative processes that lead to rapid generation of small separations with lognormal scaling in regions of high 


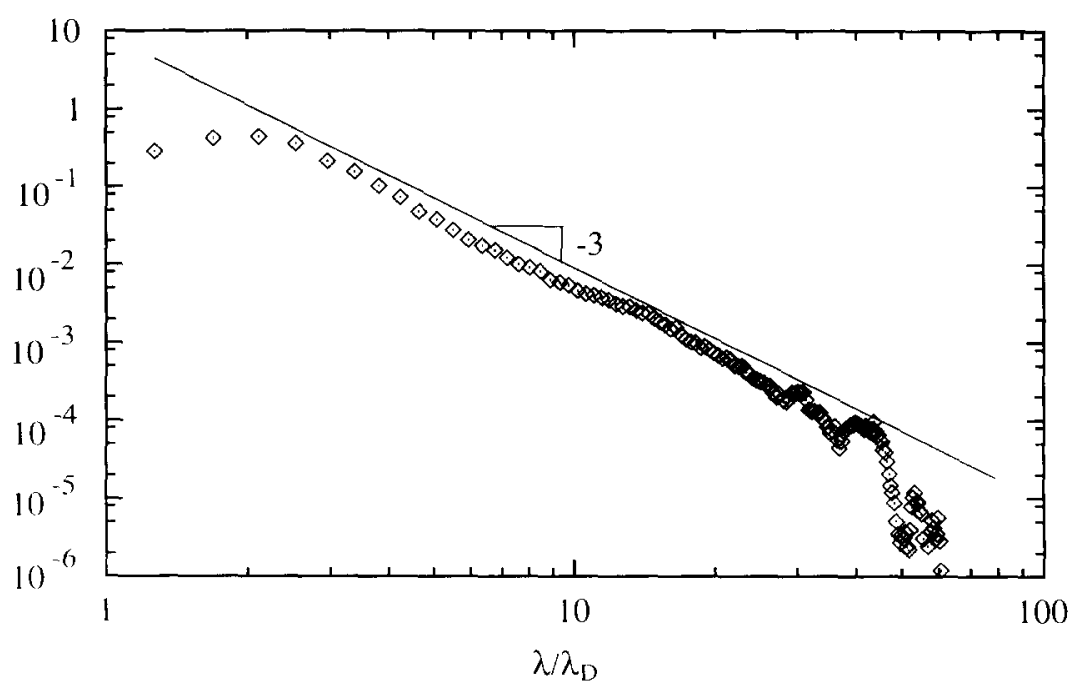

Fig. 12. Composite layer separation PDF for the fully three-dimensional turbulent shear flow data with $R e \approx 3700$. The probability density function is calculated from 29 temporally spaced volumes of which Fig. 1 is an example using the three-dimensional algorithm for finding scalar energy dissipation layer separation distances. Notice the prevalent -3 slope covering nearly all range of separations in this $\log -\log$ plot.

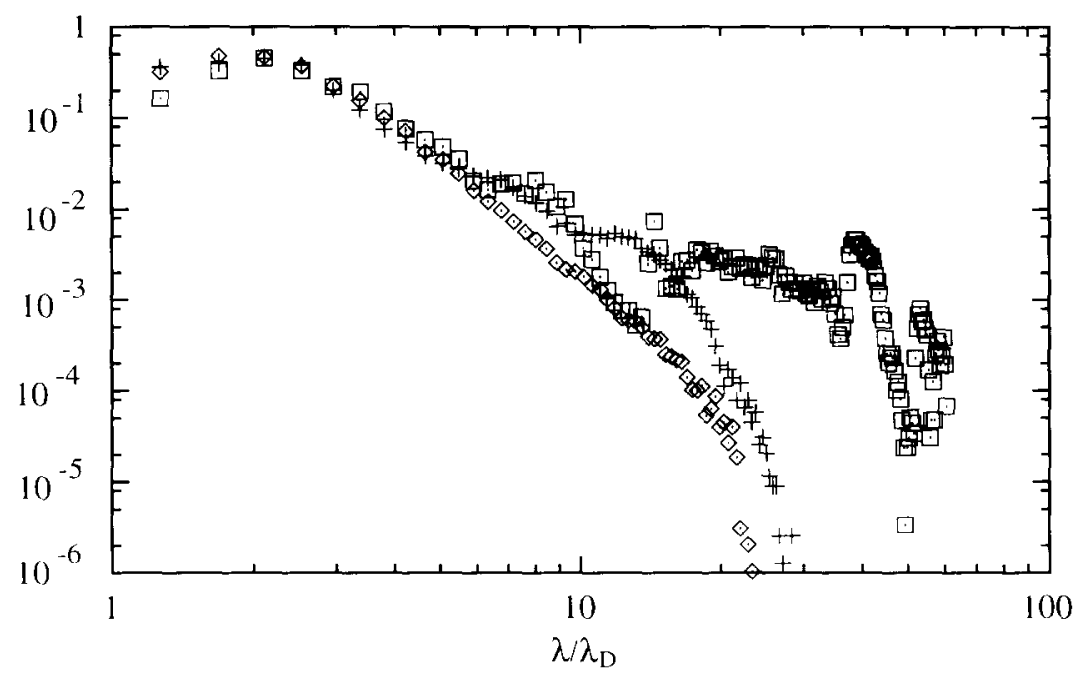

Fig. 13. Layer separation PDFs for three different volumes of the three-dimensional turbulent shear flow data of which Fig. 1 is an example. Notice how the PDFs match fairly well at small separations and can vary quite radically at large separations. This is simply consistent with the shape of the PDF which indicates that the statistics of the more prevalent smaller separation distances will settle down much faster than those of the more infrequent larger separations which will require a longer time average to obtain accurate results.

strain (large stretching values) are fairly well understood. Moreover, in the chaotic flow the presence of low stretching values leads to locally strong departures from lognormal scaling for large separations. In view of the apparent similarities in Figs 11 and 13, it might be tempting to speculate that similar dynamical processes may control the scaling characteristics of the mixing process at these scales in turbulent flows. However, there are important differences between the closed chaotic flow and the open turbulent flows. In particular, the closed chaotic flow initially requires that repeated iterations must lead to reductions in the 
dissipation layer separations. In the open turbulent flow, however, ambient fluid is continually entrained into the mixing region, creating voids in the dissipation field which are subsequently broken down by the stretching and folding process. The large separations in the turbulent flow result principally from this entrainment process, and the -3 scaling in the ensemble-averaged distribution is merely the result of the distribution of large voids created by the entrainment and their subsequent breakdown ('cascade') process. In the chaotic flow, the breakdown of the initial voids might be expected to occur by a different process, leading to a different scaling of the dissipation layer separations.

Despite these arguments, the similarities in Figs 11 and 13 are sufficiently striking to motivate a comparison of the ensemble-averaged distributions in Figs 9 and 12. The result is shown in Fig. 14, and reveals that the scaling of the layer separation distributions in the chaotic flow and the turbulent flow agree remarkably well. Following the reasoning given above, the apparently common lognormal scaling for small $\lambda$ is not surprising. Moreover, any similarity in the value of $\lambda$ at which the crossover from lognormal scaling to the -3 power law scaling might appear to occur is likely only coincidental, since in the chaotic flow this crossover location will depend on the iteration number $n$ (see Fig. 3(b) of ref. [13]). Nevertheless, based on the above arguments there is no apparently obvious reason why the two flows should have the same scaling at large $\lambda$ values. In the chaotic flow the scaling of the layer separation distribution in this range of length scales appears to be dominated by the persistence of slow processes near the voids. In the turbulent flow, if these large separations are in fact dominated by the entrainment process, then there would seem to be little prospect of any dynamical similarity at these scales between these two types of flows. On the other hand, given the relatively small size of cubes like those in Fig. 1 relative to the local outer scale $\delta$, it seems likely that initially large voids created by the entrainment process have already undergone many periods of a repeated breakdown process, and thus might be more reflective of the dynamics of the small scales of turbulence than of the entrainment process itself. If that is the case, then the similar scaling in Fig. 14 for large values of $\lambda$ leaves open the possibility of some dynamical similarity between the

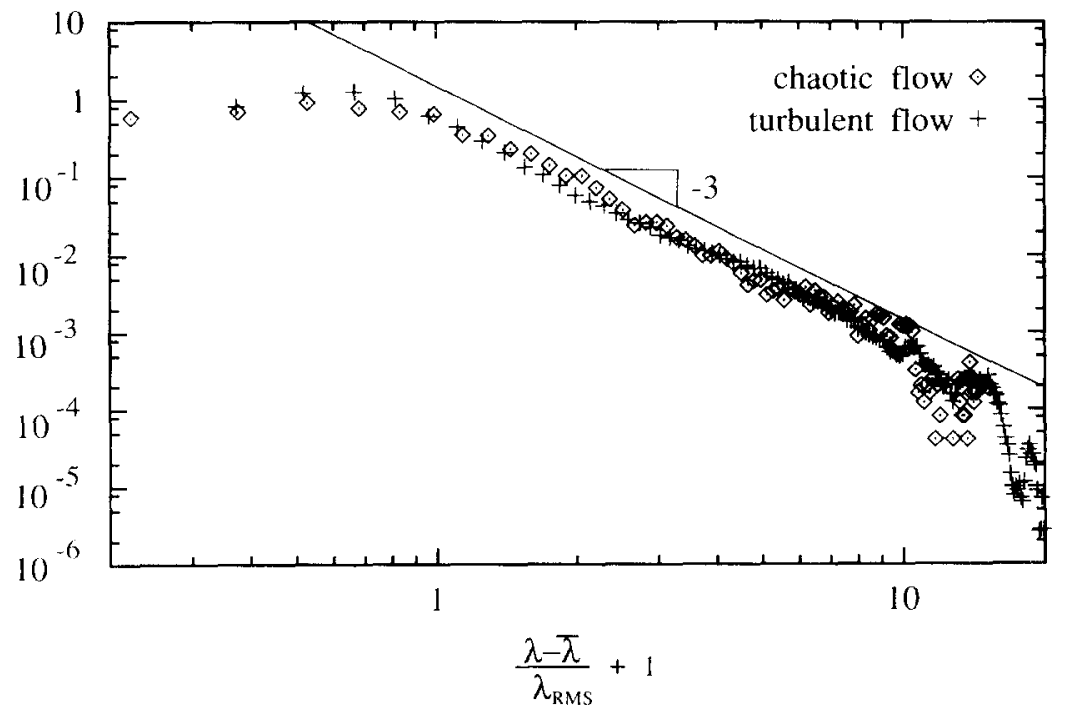

Fig. 14. Overlay of composite PDFs of layer separations for both the chaotic flow of Fig. 2. and the turbulent flow of Fig. 1. Results are presented as shown in terms of their respective first and second moments revealing a common shape between the PDFs of the two-dimensional chaotic flow and the three-dimensional turbulent flow. Notice again the -3 slope prevalent throughout nearly the entire range of scales. 
mixing produced by the small scales of turbulent flows and by the chaotic flow. This possibility cannot be argued for strongly on the basis of the results presented here. For the present time we simply note that the similarities evident in Fig. 14 in the mixing signatures of these chaotic and turbulent flows are interesting, and may only be coincidental, but are certainly unexpected.

\section{FRACTAL SCALING PROPERTIES}

The suggestion of potential fractal scaling in various geometric features of high Reynolds number turbulent flows can be traced back to the oldest descriptions of turbulence. Some of the earliest direct experimental evidence for fractal scaling of mixing in turbulent flows was reported by Sreenivasan and Meneveau [19] from single-point measurements of a conserved scalar field (temperature) in the turbulent wake of a slightly heated body. These findings eventually led to further evidence of fractal geometric scaling in isosurfaces of a conserved scalar quantity from two-dimensional imaging measurements in the self-similar far field of an axisymmetric turbulent jet [20,21]. Subsequently, Miller and Dimotakis [22] conducted highly-resolved single-point measurements of conserved scalar mixing in a turbulent jet, and reported finding fractal scaling for only a very narrow range of isoscalar values, with distinctly non-fractal scaling for other threshold values. More recently, Lane-Serff [23] has reported two-dimensional imaging measurements of scalar mixing in turbulent jets that appear to support the suggestion that geometric scaling properties of isosurfaces in turbulent mixing can depend significantly on the chosen isoscalar value. Though it is possible that the geometry that results from mixing in turbulent flows might actually differ fundamentally for differing isoscalar surfaces, this runs counter both to intuition and classical scaling concepts for turbulent flows. Instead, it could be argued that the apparent differences in geometric properties of various isoscalar surfaces might be more directly an artifact of the experiments than of the flow, since a small change in the isoscalar value typically produces a rather significant change in the geometry of the isoscalar contour.

By contrast, owing to its compact nature, the geometry of the support of the scalar dissipation field in Fig. 1 should be relatively insensitive to the choice of dissipation threshold level. This suggests an assessment of the potential fractal scaling properties of mixing in turbulent flows from the perspective of the scalar dissipation field rather than the scalar field. In this section, we present a comparison of the applicability of fractal scaling concepts for the geometry of the scalar dissipation field in chaotic and turbulent flows. Although data of the type in Figs 1 and 2 offer access to the fully three-dimensional scalar dissipation rate field, this comes at the expense of a limited spatial dynamic range in the data. Unlike long time-series data, spatial data of this type offers only a limited number of pixels in any given direction from which to assess a potential fractal scaling. This in turn requires novel methods for assessing the geometric scaling properties on which to base a judgement of the applicability of fractal scaling concepts. Accordingly, in Section 4.1 we describe the statistical box counting scheme used here to calculate the local scaling dimension of linear intersections through the three-dimensional dissipation fields. Following this, Sections 4.2 and 4.3, respectively, present results obtained for the geometric scaling properties of the scalar dissipation support in the chaotic and turbulent flows.

\subsection{Fractal measurement method}

In both the chaotic and turbulent flows, we compute the local scaling dimensions of one-dimensional intersections with the support set on which the three-dimensional scalar 
dissipation field lies above some chosen threshold value. Owing to the inherently short record lengths available for tiling, a statistical assessment of the geometric scaling properties was used. Briefly, for any given one-dimensional intersection, the tiling method used for computing the local scaling properties is a variation of the conventional box counting scheme. The tile size $\delta$ at different tiling levels was varied by factors of two. The tiling procedure used several different initial tile locations (offsets) to remove bias that results from a fixed starting point. The two partial tiles created at both ends of the domain as a result of the offsets were counted together as one tile. This tiling algorithm for one-dimensional intersections was tested on a wide range of both regular and irregular Cantor sets of various known fractal dimensions to assess its suitability for accurately dealing with short record lengths. In each case, the true fractal dimension $D$ was computed via

$$
\sum_{i=1}^{n} c_{i}^{D}=1
$$

where $c_{i}$ is the length ratio of the $i$ th segment in the generator to the total length of the initiator, and $n$ is the total number of segments in the generator. The total number of tiles required to cover the set at any scale $\delta$ is $N(\delta)$. Typical results obtained for dense Cantor sets with true fractal dimensions of $0.8,0.6,0.4$, and 0.2 are given in Fig. 15. In each case, the figure shows the local fractal dimension (LFD) obtained at any tile size $\delta$, defined as $-d \log _{2} N(\delta) / d \log _{2} \delta$, giving a sensitive measure of the local scaling properties. The LFD curves in Fig. 15 were computed via linear central differences of $\log _{2} N(\delta) / \log _{2} \delta$ curves of the type shown in Fig. 16. Note that simply least squares fitting a straight line through data such as in Fig. 16 assumes that fractal scaling holds for the given set, while LFD curves like those in Fig. 15 provide a means for independently assessing the validity of a fractal scaling for the set.

The LFD curves in Fig. 15 demonstrate a number of features essential for this study. First, it should be noted that, for both regular and irregular Cantor sets, for $D \geqslant 0.4$ the box-counting algorithm accurately converges to the true dimension for any one realization of the fractal as the tile size $\delta \rightarrow 0$. In other words, if a sufficiently large number of data points are contained in any one-dimensional intersection, then the present algorithm is capable of accurately determining the fractal dimension of that intersection. However, for low fractal dimensions, this convergence can be seen to become very slow. For $D=0.2$, for example, $2^{17}$ points are evidently not sufficient to obtain convergence to a confident assessment of the true dimension. For such low values of $D$, the fractal set is so sparce that the number of tiles $N(\delta)$ remains relatively small even when the tile size $\delta$ is made very small. As a consequence, the \pm 1 tile ambiguity inherent in any tiling scheme leads to a non-trivial fractional change in $N(\delta)$, and thus upon differentiation to a large change in the LFD (see Fig. 16). This will remain the case until $\delta \rightarrow 0$ as $D \rightarrow 0$. This limit is generally more forgiving for irregular Cantor sets than for regular sets, but for any minimum tile size $\delta$ there is still a limit to the minimum fractal dimension $D$ that can be reliably determined. A further phenomenon that makes determining low values of $D$ less accurate is the fact that, since low-dimensional sets occupy so little space, the same number of tiles $N(\delta)$ may suffice to cover them for more than one tile size $\delta$. This would produce an LFD value of 0 for that value of $\delta$. If infinitely many offsets were used, this effect could be removed, but any finite number of offsets will cause such imperfections to appear in the LFD curves of low-dimensional fractals.

The results in Fig. 15 also show that, whereas determining the fractal dimension via a tiling scheme is relatively straightforward for arbitrarily long records, for the comparatively short $2^{7}$-point scalar dissipation records available from our imaging measurements of the 

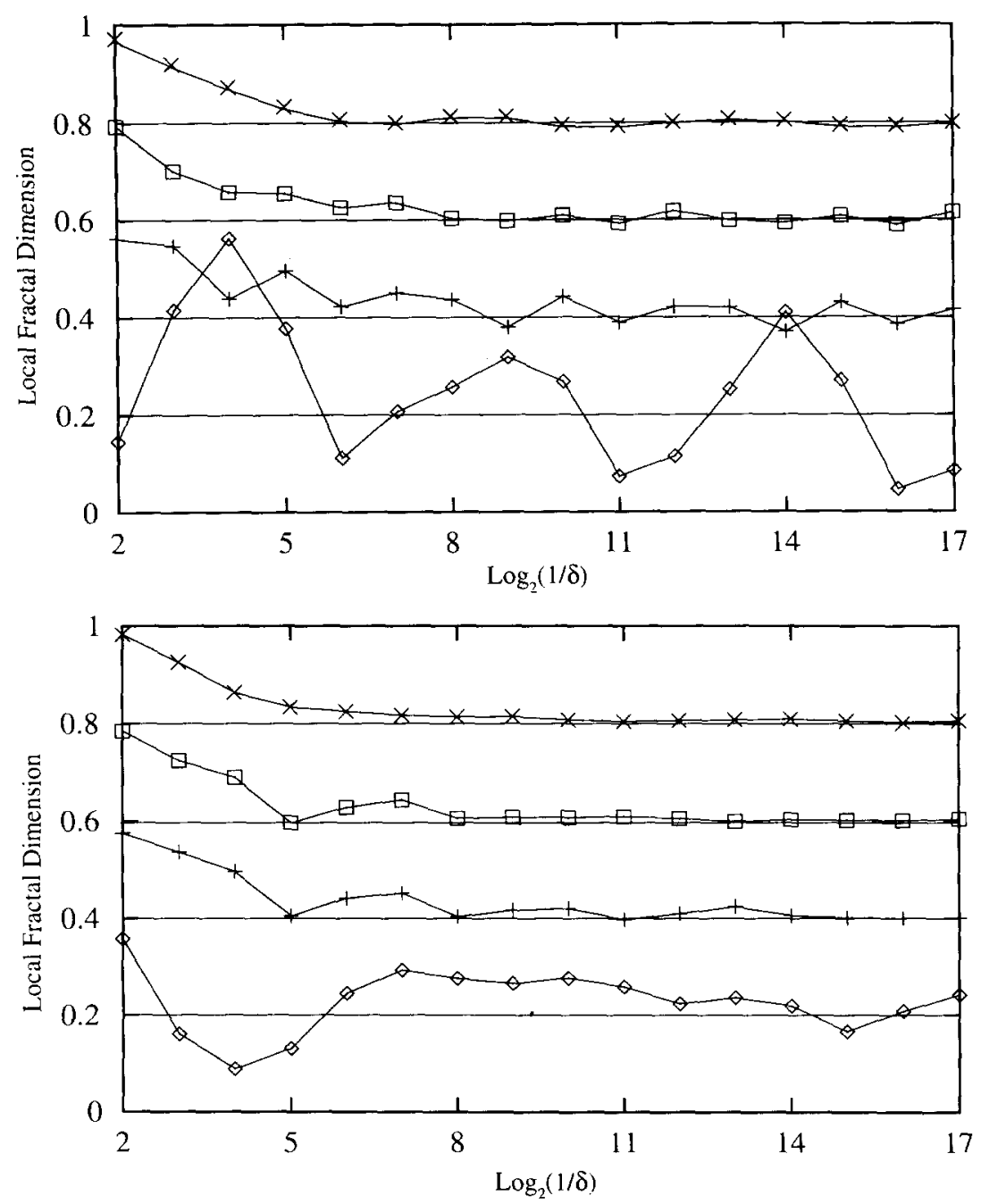

Fig. 15. Local fractal dimension curves for regular and irregular cantor sets with varying fractal dimensions, measured with the box-counting algorithm. Notice that agreement decreases dramatically with decreasing fractal dimension. In the measurements done on the chaotically advected and turbulent data sets only values in the range $2 \leqslant \log _{2}(1 / \delta) \leqslant 7$ will be available. (a) Regular cantor sets; (b) irregular cantor sets.

chaotic and turbulent flows, finding the fractal dimension is anything but straightforward. In particular, any direct assessment of the dimension on the basis of LFD plots such as those in Fig. 15 up to $\log _{2} \delta \leqslant-7$ is out of the question for all but the highest values of $D$. For $\log _{2} \delta \leqslant-4$, it is evident that the tiles are too coarse to provide any useful scaling information. For our short records, this leaves the range $-4 \leqslant \log _{2} \delta \leqslant-7$ for assessing the potential fractal scaling properties of the dissipation support set, and for determining its dimension if it is fractal.

However, we can recognize that the fluctuations in the LFD curves in Fig. 15 for any given value of $D$ result from idiosynchracies of that one particular realization of the Cantor set. Since in general each realization of the fractal will be similar only in its scaling properties and will differ in its precise details, we can anticipate that the fluctuations will differ from one realization to another. This motivates a statistical approach as indicated in 


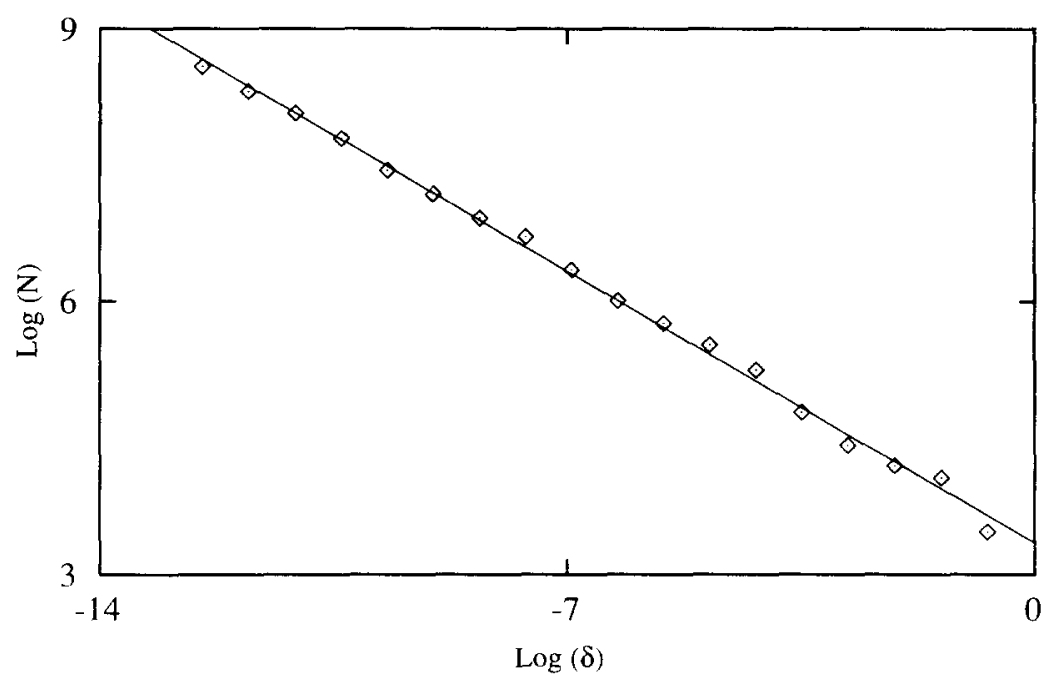

Fig. 16. A plot of the logarithm of the number of cells vs the logarithm of the cell size of an irregular cantor set with $D=0.40$. Comparing this with the local fractal dimension curve in Fig. 15(b) shows that the local fractal dimension curves are visually more sensitive to non-fractal behavior.

Figs 17 and 18. In particular, for many realizations of any one fractal with given dimension $D$, we can collect statistics of the LFD value over the range $-4 \leqslant \log _{2} \delta \leqslant-7$ to construct the probability density of LFD values. Note that, if it were possible to take $\delta \rightarrow 0$, the variance $\sigma$ of this distribution would also tend to zero, and a direct assessment of $D$ would be possible. Constrained to the range of $\delta$ achievable with our short record lengths, we can nevertheless determine $\langle D\rangle$ and $\sigma^{2}(\langle D\rangle)$ as indicated from a large number of independent realizations of known fractal sets over this range of $\delta$. The resulting $\sigma^{2}(\langle D\rangle)$ then provides the measure by which a given set can be determined to be fractal or non-fractal. For a given set, its $\langle D\rangle$ and $\sigma^{2}$ can be determined as in Figs 17 and 18, and then compared against the ideal $\sigma(\langle D\rangle)$ generated from perfect fractals using irregular Cantor sets. If the measured variance is comparable to the ideal variance for the measured value of $\langle D\rangle$, then the set 'is as fractal as a true fractal is' over this range of tile sizes $\delta$.
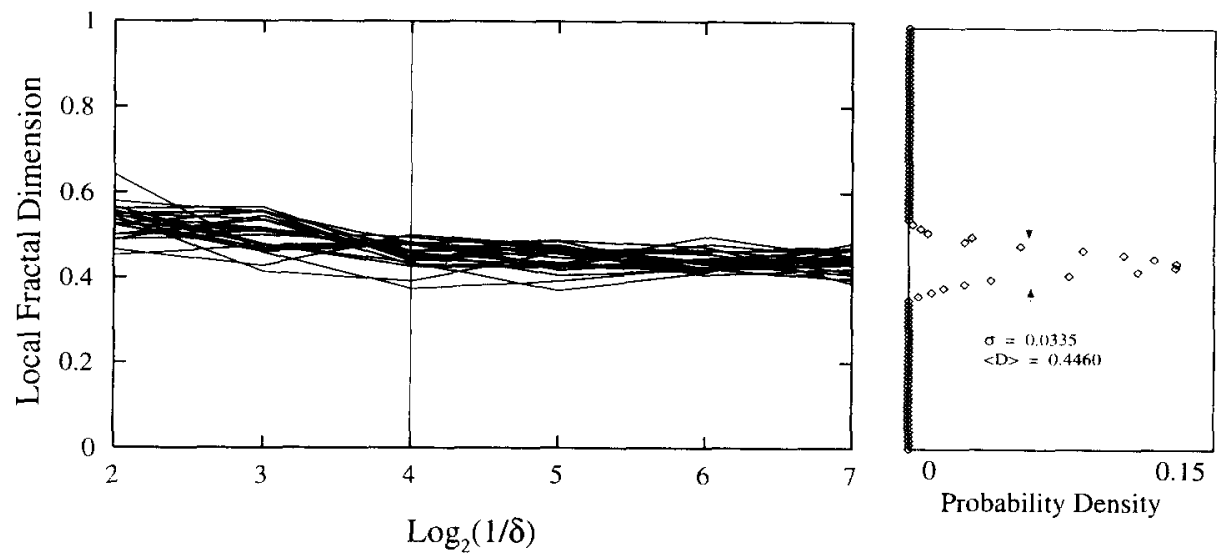

Fig. 17. Sample local fractal dimension curves from among the 8000 irregular cantor sets generated as test cases (dimension $=0.40$ ) along with a cumulative probability density curve for all of the local fractal dimension values in the range $4 \leqslant \log _{2}(1 / \delta) \leqslant 7$. 
It thus becomes essential to accurately tabulate $\sigma^{2}(\langle D\rangle)$ for genuine fractal sets. To do this, the tiling algorithm described above was repeated for 8000 individual independent realizations of irregular Cantor sets each having the same fractal dimension $D$. This was repeated for each of nine different fractal dimensions between 0.1 and 0.9 (Table 1).

Note that the slight increase in $\sigma^{2}(\langle D\rangle)$ for $D=0.9$ is probably due to the difficulty in applying the generator for high-dimensional Cantor sets until the appropriate resolution is reached. In order to make a set with high dimension and the appropriate resolution in a reasonable number of generator applications, a limit had to be placed on the maximum $c_{i}$. This restricted the details of these high-dimensional sets, which could affect the convergence of the LFD statistics. Note also that, in addition to the variance $\sigma^{2}(\langle D\rangle)$, local probability density plots of the type shown in Fig. 18 provide an additional and somewhat more detailed signature of a true fractal, against which analogous results for a given set can be compared to assess the applicability of fractal concepts to its scaling properties.

An assessment of the possible effects of noise in the original scalar field measurements on the scaling properties of the resulting scalar dissipation support set was conducted via the following procedure. First, an approximate value of the r.m.s. noise in the dissipation fields was obtained by measuring the r.m.s. noise found in uniform regions of the original scalar fields and applying classical noise estimation techniques. Next, a Gaussian noise distribution with the same r.m.s. value was added to the scalar energy dissipation rate fields, which were then thresholded and compared with the original thresholded scalar energy dissipation rate fields. By comparing the original fields to the noisy fields, probability density functions were calculated giving the likelihood of a given pixel being turned on or off due to noise, depending on the distance from the nearest point on the original noise-free set. This procedure was used to add noise to an irregular Cantor set and LFD curves generated as described above. The results in Fig. 19 show that even noise levels much higher than those in either set of experimental data appear to have little effect on the measured LFD curves.

\subsection{Chaotic flow results}

In the chaotic flow, LFD curves were generated for each non-empty row and column in the $256 \times 256$ spatial data planes in the two-dimensional scalar dissipation field. Statistics were collected in each window shown in Fig. 2. There were many individual rows and columns that exhibited fractal scaling properties, i.e. for which $\sigma(\langle D\rangle)$ was within the values in Table 1 over the range $-4 \leqslant \log _{2} \delta \leqslant-7$ (see Fig. 20). However, many other one-dimensional intersections displayed a scaling that could not be remotely termed fractal

Table 1. Statistics of known fractal sets collected over the range $-4 \leqslant \log _{2} \delta \leqslant-7$ for many different realizations via the procedure outlined in Figs 17 and 18.

\begin{tabular}{ccc}
\hline$D$ & $\langle D\rangle$ & $\sigma^{2}(\langle D\rangle)$ \\
\hline 0.1 & 0.199 & 0.065 \\
0.2 & 0.233 & 0.064 \\
0.3 & 0.333 & 0.052 \\
0.4 & 0.446 & 0.036 \\
0.5 & 0.543 & 0.026 \\
0.6 & 0.638 & 0.023 \\
0.7 & 0.733 & 0.021 \\
0.8 & 0.830 & 0.020 \\
0.9 & 0.930 & 0.021 \\
\hline
\end{tabular}




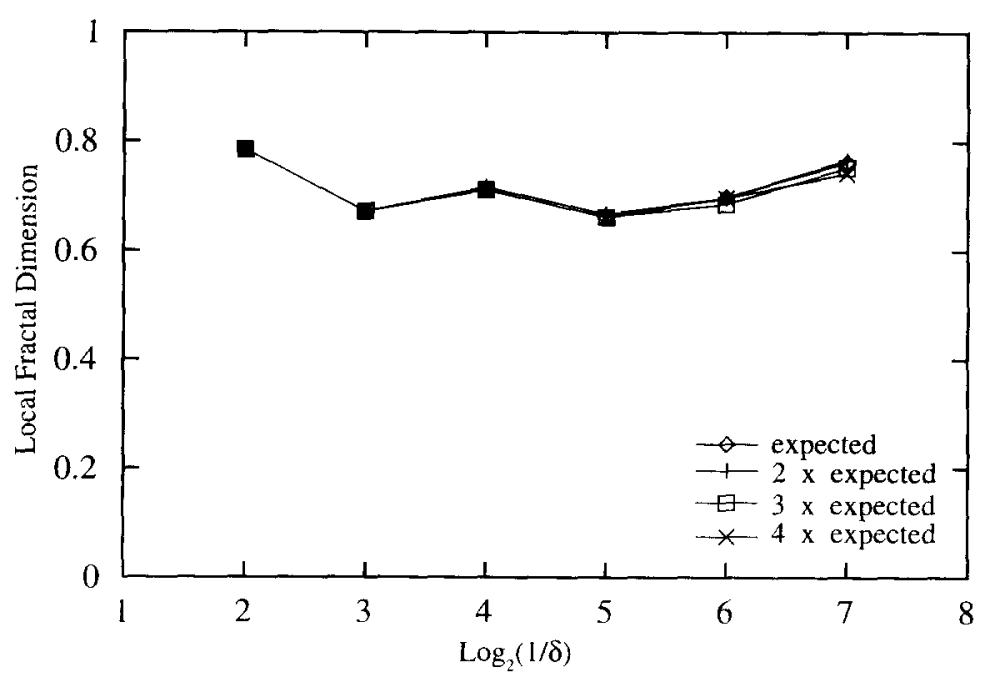

Fig. 19. The effect of differing amounts of noise on the local fractal dimension curve of an irregular cantor set that has been digitized to 256 pixels. Noise does not appear to be much of a factor in influencing the local fractal dimension, even at levels much higher than those present in the experimental data.

(see Fig. 20). Notice that the differences are not as strong in the $\log N(\delta)$ vs $\log \delta$ curves in Fig. 20(a), but show up clearly in the LFD curves in Fig. 20(b). However, none of the data within an entire window produced $\sigma^{2}(\langle D\rangle)$ values that fell even within a factor of two of the values given in Table 1. Moreover, changing the dissipation threshold level over the range $1.0 \leqslant \chi /\langle\chi\rangle \leqslant 2.5$, as shown in Fig. 21 , did not produce any change in this result - no window produced a geometric scaling that came within a factor of two of the criterion in Table 1 and Fig. 18 for declaring a set as being fractal.

Figure 22 shows the local corresponding local probability density plot analogous to Fig. 18 for the ensemble-averaged statistics over all 31 windows in Fig. 2. As the figure indicates, these ensemble statistics can be confidently declared as being non-fractal.

(a)

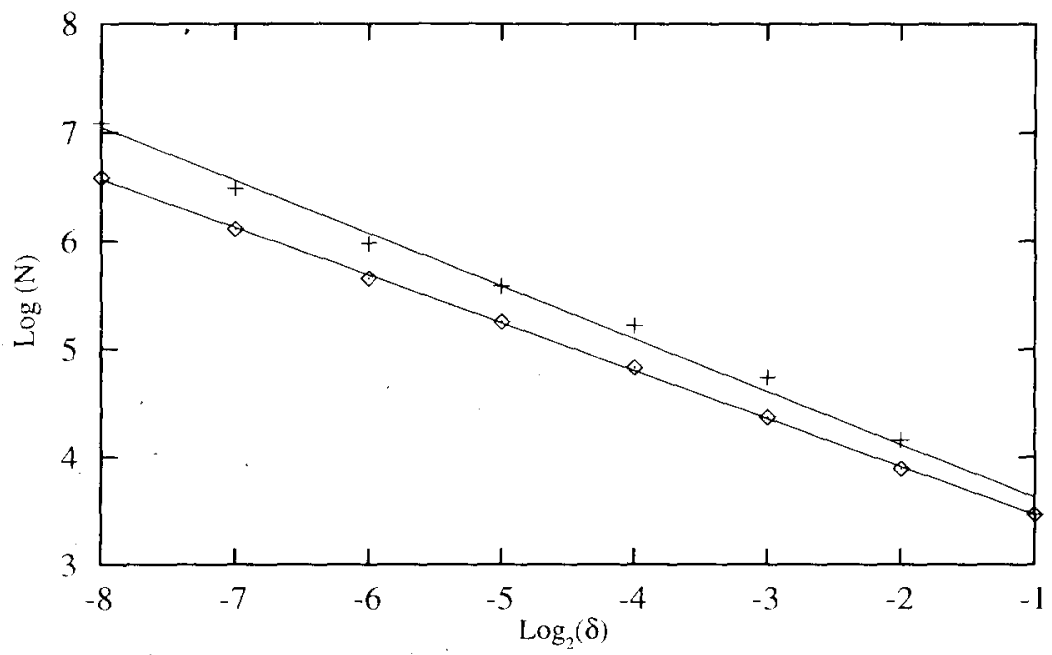

Fig. 20(a). 
(b)

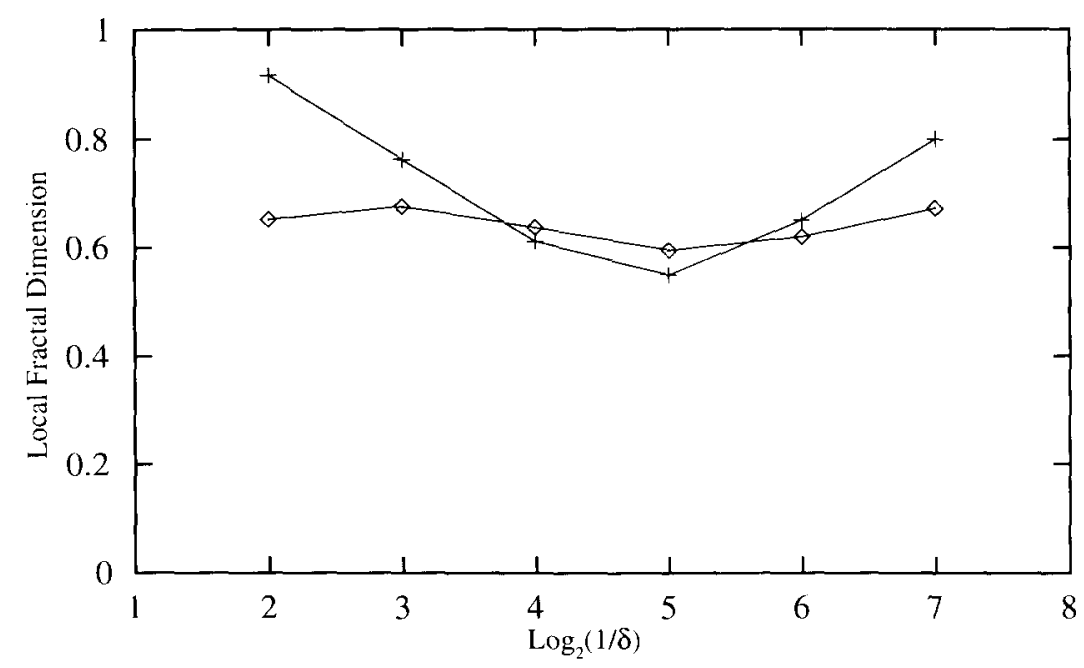

Fig. 20. Local fractal dimension curves and $\log (N)$ vs $\log _{2}(\delta)$ plots for two data samples from the chaotically advected data set. One data sample appears to be quite fractal while the other shows no sign of being fractal. Notice that the local fractal dimension curves accentuate any deviations from fractal behavior. (a) Log $(N)$ vs $\log _{2}(\delta)$ plot. (b) Local fractal dimension curves.

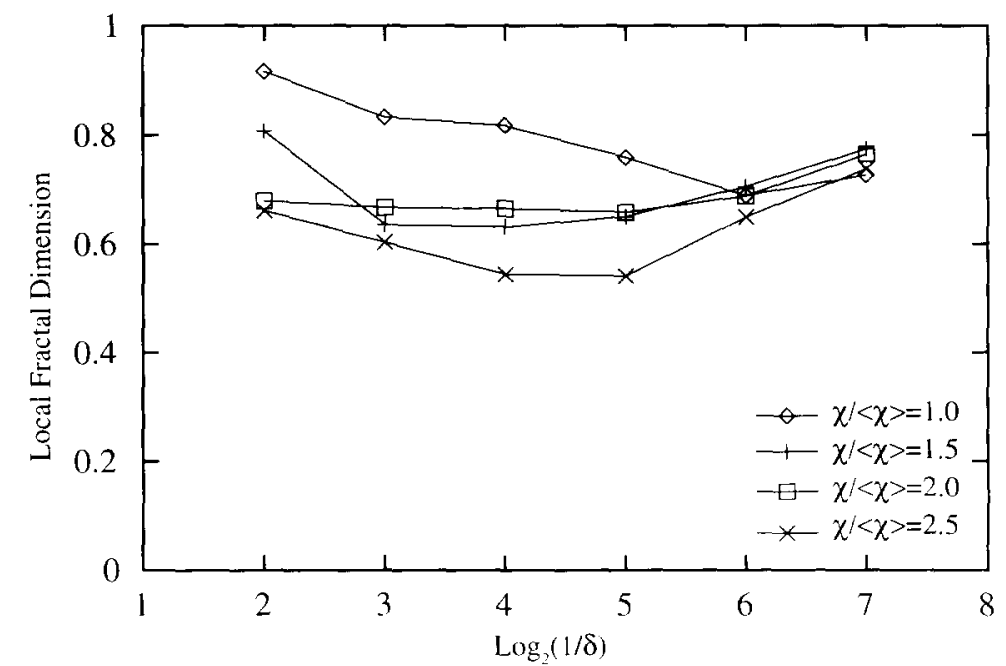

Fig. 21. Threshold level effects on a representative data sample from the chaotically advected data set are shown above. It appears that in general the results are relatively threshold-independent considering that the threshold value varies by a factor of 2.5 .

However, within 'parallel' regions of the flow, much tighter LFD probability distributions were found. An example is shown in Fig. 23, corresponding to a region in which essentially all the dissipation layers were parallel, and which produced an essentially lognormal layer separation distribution in Section 3. Even in this case, however, the $\sigma^{2}(\langle D\rangle)$ value lies well above that given in Table 1 for the same $\langle D\rangle$. This can be seen by comparing Figs 18 and 23, and keeping in mind that the width of the LFD distributions in Table 1 decrease with increasing $D$. Lastly, Fig. 24 shows an LFD map for a window corresponding to a 'non parallel' region of the flow. In this case, the variance is nearly five times that given in 
Table 1, and the scaling can be confidently said to be non-fractal. Generally speaking, the most nearly fractal regions of the flow seemed to coincide with the areas in which the layers were parallel, while the clearly non-fractal regions coincided with intermediate and non parallel regions. However, at this stage of development of the flow, no regions led to a scaling sufficient to declare the dissipation support as being globally fractal.

\subsection{Turbulent flow results}

For the turbulent flow, analogous LFD curves were generated for each non-empty row and column in the $256^{3}$ spatial data volumes in the three-dimensional scalar dissipation field. As was the case in the chaotic flow results, many individual one-dimensional intersections of the turbulent flow data exhibited nearly fractal scaling (e.g. see Fig. 25).

(a)

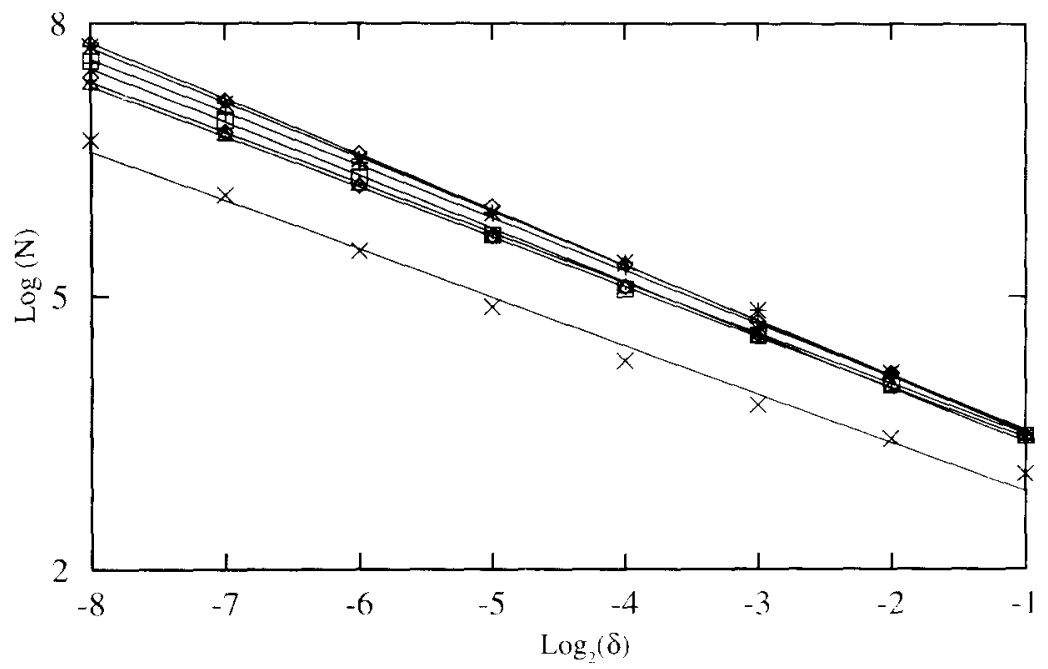

(b)

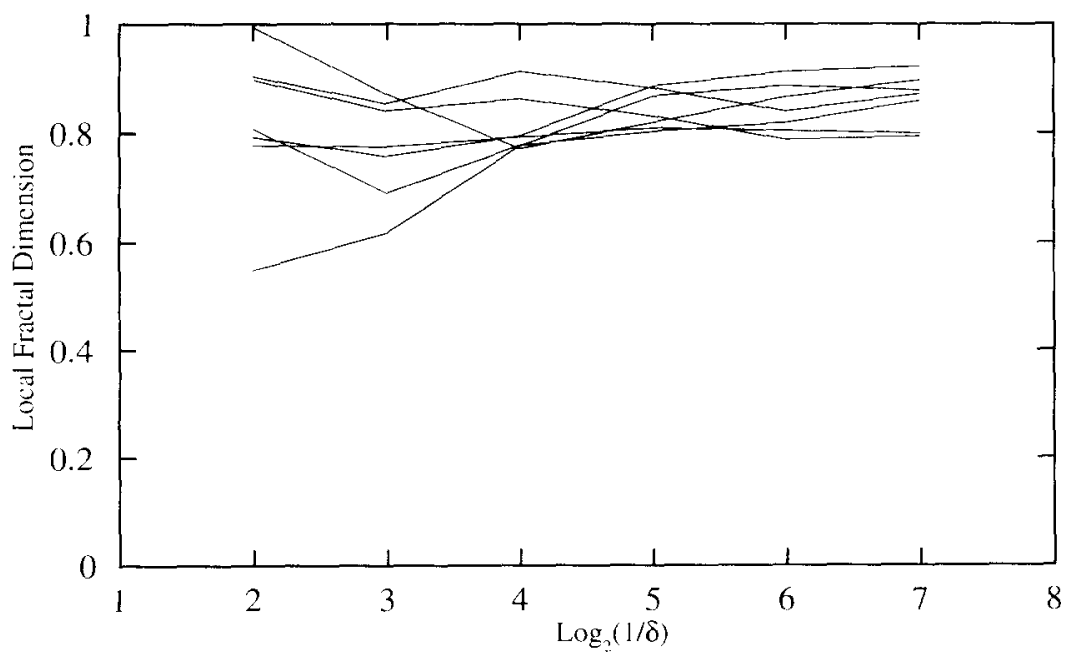

Fig. 25. Local fractal dimension curves and $\log (N)$ vs $\log _{2}(\delta)$ plots for seven data samples from the turbulent data set. Some data samples once again appear to be quite fractal, while others show no sign of being fractal. (a) $\log (N)$ vs $\log _{2}(\delta)$ plot; (b) local fractal dimension curves. 


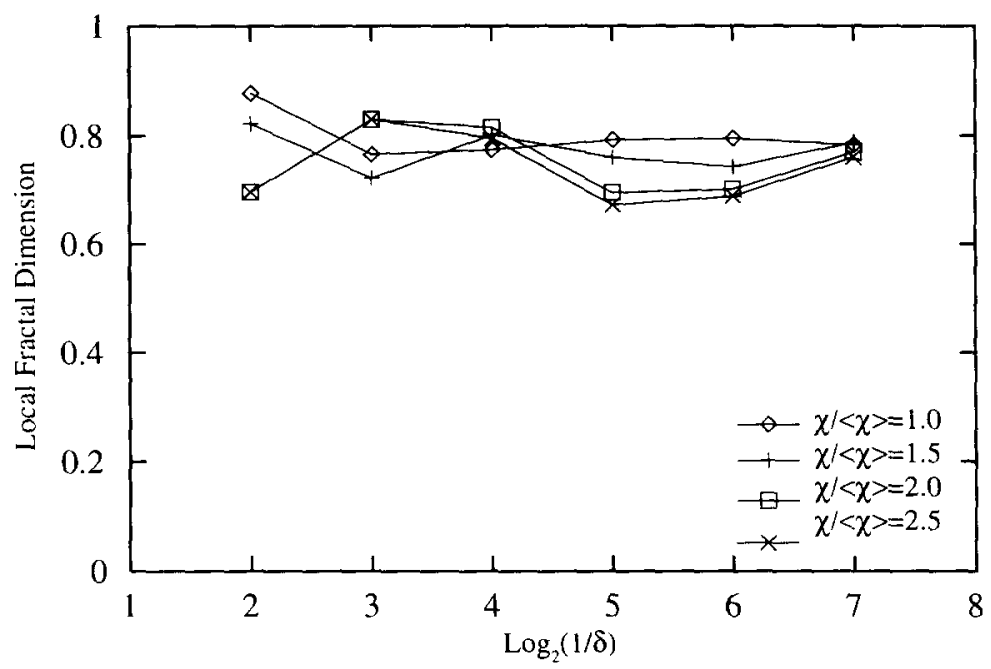

Fig. 26. Threshold level effects on a representative data sample from the turbulent data set are shown above. It appears that, in general, the results are relatively threshold-independent considering that the threshold value varies by a factor of 2.5 .

Moreover, the LFD curves for the turbulent flow were found to be largely independent of the $\chi /\langle\chi\rangle$ threshold values over the range from 1.0-2.5, as shown for typical cases in Fig. 26. Nevertheless, when local probability density plots were constructed for typical regions in the turbulent flow data (e.g. Fig. 27), the resulting $\sigma^{2}(\langle D\rangle)$ values were all well above those in Table 1 . Even for the most nearly fractal regions, the measured $\sigma^{2}(\langle D\rangle)$ based on these one-dimensional intersections was still approximately three times higher than in Table 1. Generally speaking, in the turbulent flow data there was much less variation in the results among different regions. This was presumably due to the fact that there were no large regions in the data where all the scalar energy dissipation layers were all parallel to each other. Throughout most of the turbulent flow data, the dissipation layers were highly convoluted and tended to fold back on themselves many times in a single region. Collectively, the present results do not support a contention that the scalar dissipation rate field in turbulent flows display a global fractal scaling over the range of length scales accessible by our data.

\section{CONCLUSIONS}

The results presented here have compared two rather sensitive statistical geometric signatures of the mixing process at the small scales of high Reynolds number: threedimensional, highly unsteady, open turbulent flows with those in low Reynolds number and two-dimensional, time-periodic, closed chaotic flows. In each of these flows, measurements of the mixing of a dynamically passive, conserved scalar field $\zeta(\mathbf{x}, t)$ by the underlying flow field were used to analyze geometric scaling properties of the support set on which the associated scalar energy dissipation rate field $\nabla \zeta \cdot \nabla \zeta(\mathbf{x}, t)$ was concentrated. The results from the present one-dimensional intersections are not consistent with a global fractal description, having a single fractal dimension, for the geometric scaling properties of the scalar dissipation support set in this turbulent flow. In the chaotic flow, however, the present results are essentially consistent with a fractal description of the scaling in those regions of the flow that have undergone sufficient stretching and folding. Perhaps most 
interesting is the observation that the distribution of dissipation layer separations in both the chaotic and turbulent flows follow the same scaling over a wide range of length scales. This suggests the possibility that similar dynamical features may play a role in the mixing process in both the chaotic and the turbulent flows. It may even be possible that the classical power law scaling characteristics of the small scales in turbulent mixing may be usefully approached from this novel perspective.

Despite the attraction of working in terms of the full three-dimensional scalar dissipation rate field rather than the scalar field itself (for both practical and dynamical reasons), the results obtained here must be viewed cautiously. At a minimum, the Reynolds numbers in the turbulent flow data are large enough for a highly unsteady and apparently 'turbulent' mixing process to result, but are still well below the range of values traditionally considered safe for statements of high Reynolds number asymptotic behavior. However, our attention here has been focused on the small scales of the turbulent mixing process. If these are governed largely by the local dynamics of the continual stretching and folding of material elements that results from the time-varying strain rate and vorticity in the underlying velocity field, then traditional requirements for Reynolds number asymptotics, such as an extensive inertial range, may be relatively unimportant. While it would certainly be interesting to look for additional Reynolds number scaling and asymptotic limits in measures of the mixing dynamics such as those considered here, such high Reynolds number studies are not possible with presently available experimental techniques. Moreover, in view of the dynamical similarities of the stretching and folding processes at the small scales of turbulent flows and in chaotic flows, it may be more important to have access to fully three-dimensional dissipation field data, as we have done here, than to consider lower-dimensional estimates of the mixing process at higher Reynolds number values.

Acknowledgements - This work is supporting the program in Turbulence Structure and Control at the Air Force Office of Scientific Research (AFOSR) through AFOSR Grant No. F49620-92-J-0025. Dr. J. M. McMichael is the program manager. We are grateful to Julio Ottino for providing us with the original photonegatives of his eccentric-cylinder flow experiments, and to Lester Su for assistance in development of the layer center detection algorithm.

\section{REFERENCES}

1. W. J. A. Dahm, K. B. Southerland and K. A. Buch, Direct, high resolution, four-dimensional measurements of the fine scale structure of $S c \gg 1$ molecular mixing in turbulent flows, Phys. Fluids A3, 1115-1127 (1991).

2. W. J. A. Dahm, L. K. Su and K. B. Southerland, A scalar imaging velocimetry technique for fourdimensional velocity field measurements in turbulent flows, Phys. Fluids A4, 2191-2206 (1992).

3. L. K. Su and W. J. A. Dahm, Scalar imaging velocimetry measurements of the small scale structure and dynamics of fluid turbulence, Phys. Fluids A, submitted (1993).

4. J. M. Ottino, The Kinematics of Mixing: Stretching, Chaos, and Transport. Cambridge University Press, Cambridge (1989).

5. H. Aref, Integrable, chaotic, and turbulent vortex motion in two-dimensional flows, Ann. Rev. Fluid Mech. 15, 345-389 (1983).

6. H. Aref, Stirring by chaotic advection, J. Fluid Mech. 143, 1-21 (1984).

7. J. M. Ottino, C. W. Leong, H. Rising and P. D. Swanson, Morphological structures produced by mixing in chaotic flows, Nature Lond. 333, 419-425 (1988).

8. J. M. Ottino, The mixing of fluids, Sci. Am. 260, 56-67 (1989).

9. J. M. Ottino, Mixing, chaotic advection, and turbulence, Ann. Rev. Fluid Mech. 22, 207-253 (1990).

10. T. Dombre, U. Frisch, J. M. Greene, M. Hénon, A. Mehr and A. M. Soward, Chaotic streamlines in the ABC flows, J. Fluid Mech. 167, 353-391 (1986).

11. H. A. Kusch and J. M. Ottino, Experiments on mixing in continuous chaotic flows, J. Fluid Mech. 236, 319-348 (1992).

12. P. D. Swanson and J. M. Ottino, A comparative computational and experimental study of chaotic mixing in viscous fluids, J. Fluid Mech. 213, 227-249 (1990).

13. F. J. Muzzio, P. D. Swanson and J. M. Ottino, The statistics of stretching and stirring in chaotic flows, Phys. Fluids A3, 822-831 (1991). 
14. F. J. Muzzio, P. D. Swanson and J. M. Ottino, Mixing distributions produced by multiplicative stretching in chaotic flows, Int. J. Bifurcations \& Chaos 2, 37-50 (1992).

15. F. J. Muzzio, C. Meneveau, P. D. Swanson and J. M. Ottino, Scaling and multifractal properties of mixing in chaotic flows, Phys. Fluids A4, 1439-1456 (1992).

16. S. Redner, Random multiplicative processes: An elementary tutorial, Am. J. Phys. 58, $267-273$ (1990).

17. E. Ott and T. M. Antonsen, Fractal measures of passively convected vector fields and scalar gradients in chaotic fluid flows, Phys. Rev. A39, 3660-3671 (1989).

18. E. Ott and T. M. Antonsen, The spectrum of fractal dimensions of passively convected scalar gradients in chaotic fluid flows, Phys. Fluids A3, 1417-1430 (1991).

19. K. R. Sreenivasan and C. Meneveau, The fractal facets of turbulence, J. Fluid Mech. 173, 357-386 (1986).

20. K. R. Sreenivasan, R. Ramshankar and C. Meneveau, Mixing, entrainment, and fractal dimension of surfaces in turbulent flow, Proc. R. Soc. Lond. A421, 79-108 (1989).

21. K. R. Sreenivasan, Fractals and multifractals in fluid turbulence, Ann. Rev. Fluid Mech. 23, $539-600$ (1991).

22. P. L. Miller and P. E. Dimotakis, Stochastic geometric properties of scalar interfaces in turbulent flows, Phys. Fluids A3, 168-177 (1991).

23. G. F. Lane-Serff, Investigation of fractal structure of jets and plumes, J. Fluid Mech. 249, 521-534 (1993). 